\title{
On Unstable Principal Bundles over Elliptic Curves
}

\author{
By \\ Stefan Helmke* and Peter Slodowy**
}

Contents

Introduction

$\S 1$. General Results

$\S 2$. Automorphism Groups

$\S 3 . \quad$ Degenerations

$\S 4$. On the Structure of Levi Subgroups

$\S 5$. Simply Connected Simple Groups

$\S 6$. Non-Simply Connected Simple Groups

References

\section{Introduction}

This article is an outgrowth of a more comprehensive project to provide a detailed account for the results announced in [13], [14]. In these reports a relation between simple elliptic singularities in the sense of Saito [22] and certain holomorphic Kac-Moody loop groups $\hat{\mathcal{L}} G$ is established which generalizes a well known theorem of Brieskorn [6], cf. also [26], relating simple singularities of type $A_{\ell}, D_{\ell}, E_{\ell}$ and the corresponding simple algebraic groups $G$.

An important ingredient in the derivation of Brieskorn's result is the orbital geometry of the unipotent variety $\mathfrak{U}(G)$ of $G$, especially in the neighborhood of a subregular unipotent orbit.

In our generalization, the unipotent variety $\mathfrak{U}(G)$ is replaced by an 'unstable' variety $\mathfrak{U}(\hat{\mathcal{L}} G)$ in $\hat{\mathcal{L}} G$ whose orbital geometry can be described, essentially

Communicated by K. Saito, April 5, 2000.

2000 Mathematics Subject Classification(s): 14H60, 14B07, 20G20.

* Research Institute for Mathematical Sciences, Kyoto University, Kyoto 606-8502, Japan.

** Mathematisches Seminar der Universität Hamburg, D-20146 Hamburg, Germany. 
due to an observation by E. Looijenga, in terms of unstable principal $G$-bundles over elliptic curves.

The main purpose of this article is a classification of the unstable bundles which enter into our analogue of Brieskorn's constructions, i.e. so-called regular and subregular ones. Whereas our primary interest lies in the case of bundles with simply connected structure group $G$, in particular those of types $D_{5}$, $E_{6}, E_{7}, E_{8}$, we have here also investigated the case of non-simply connected simple structure groups $G$. They provide a large variety of orbital geometries mainly related to the non-isolated singularities of type $A_{\infty}$ and $D_{\infty}$ discussed in [13], [14]. In another direction, we provide detailed information on the automorphism groups of many other low-codimensional bundles (i.e. associated to arbitrary maximal parabolic subgroups) which is not directly relevant for our classification.

Apart from our envisaged geometrical applications, the results of this paper may be of independent interest. For example, regular unstable $G$-bundles are of importance in the work of Friedman-Morgan-Witten on moduli spaces of semistable $G$-bundles, [9], [10], where one finds a construction of the regular unstable $G$-bundles for all simply connected $G$. Our computations also give an idea of the complexities involved in the approach of Atiyah-Bott to the cohomology of moduli spaces of $G$-bundles over arbitrary Riemann surfaces [2]. Finally, our generalization of Brieskorn's Theorem is based on the orbital geometry of holomorphic Kac-Moody loop groups. By a kind of Floquet-theory, this geometry is the same as that for co-adjoint orbits of 2-toroidal or elliptic Lie algebras ([7], [8], [23]). Independently of that device, our results on $G$-bundles apply to both contexts.

Let us give an overview of the contents of this paper. In Section 1 we recapitulate fundamental results on principal $G$-bundles over elliptic curves. They are related to the canonical or Harder-Narasimhan reduction of unstable bundles and the moduli spaces of semistable bundles. We mainly refer to previous work by Atiyah-Bott [2], Schweigert [24] and Friedman-Morgan [9].

In Section 2 we investigate the automorphism groups of $G$-bundles $\xi$. For the case of semistable bundles, we can refer to recent work of Friedman-Morgan [9]. In the unstable case, we employ the canonical reduction $\xi_{L}$ of the structure group $G$ of $\xi$ to a Levi subgroup $L \subset G$, to split $\operatorname{Aut}_{G}(\xi)$ as a semidirect product of two subgroups $\operatorname{Aut}_{L}\left(\xi_{L}\right)$ and $\operatorname{Aut}_{G}(\xi)^{+}$, where the last group is unipotent and transparently determined by the Atiyah-Bott type $\mu(\xi)$ of $\xi$. The dimension of the second group may also be viewed as the codimension of the Atiyah-Bott stratum of type $\mu(\xi)$ whereas the dimension of the first one 
yields the number of internal moduli of that stratum. We are able to effectively determine $\mu(\xi)$ in case $L$ is a proper maximal Levi subgroup of $G$.

Smaller Levi subgroups can be dealt with by means of degenerations (Section 3). The computation of $\operatorname{Aut}_{L}\left(\xi_{L}\right)$ still relies on a precise description of the structure of $L$. This is given in Section 4 , in particular with respect to the occuring fundamental groups.

In Section 5, we assume that $G$ is simple and simply connected, and we tabulate the minimal dimensions, split as the sum of the two relevant summands, of the automorphism groups of unstable $G$-bundles associated to maximal Levi subgroups. As a searched-for by-product, we obtain the classification, in particular the existence, of regular and subregular unstable $G$-bundles in all cases.

Section 6 addresses the analogous problems for non-simply connected groups. Here, the methods and results are quite involved, and we have to resort to a somewhat tedious case-by-case description. Also here, we exhibit and classify regular and subregular unstable bundles in all cases.

In view of the complications involved in the determination of the dimension of $\operatorname{Aut}_{L}\left(\xi_{L}\right)$, we should point out that our classification of regular and subregular unstable bundles essentially requires only the dimension values of $\operatorname{Aut}_{G}(\xi)^{+}$which are easily computed from the Atiyah-Bott type $\mu(\xi)$. We have pursued a complete determination of all appearing dimensions for its own sake.

Work on these as well as on the more far-reaching questions discussed in [13], [14] was started in the Spring of 1998, when the second named author was a guest at RIMS, Kyoto University. It was continued at the occasion of further encounters at RIMS, and its completion benefitted greatly from a one-year-guest-professorship of the second author at the Kitami Institute of Technology, Hokkaido, 1999-2000. We would like to thank both institutions, and especially K. Saito, Kyoto, and H. Yamada, Kitami, for their continuous moral and material support.

\section{§1. General Results}

The purpose of this section is to review basic properties of holomorphic principal $G$-bundles over a complex elliptic curve $E$ where $G$ is a complex, connected, reductive group.

\section{$\S 1.1$. Topology}

Before studying the finer analytic aspects of bundles over $E$, let us quickly recall some basic facts related to the topology of these bundles. Any holomorphic $G$-bundle $\xi$ may be regarded as a topological $G$-bundle. Such bundles are 
easily classified by an element $\gamma(\xi)$ in $H^{2}\left(E, \pi_{1}(G)\right) \cong \pi_{1}(G)$ where $\pi_{1}(G)$ is the fundamental group of $G$, (cf. [2, Section 6], or [20]). We shall call $\gamma(\xi)$ the topological type or topological class of $\xi$. It is functorial with respect to extensions $G \longrightarrow G^{\prime}$ of the structure group.

Let us have a closer look at $\pi_{1}(G)$. For details on the structure theory of reductive groups we refer, here and in the sequel, to the textbooks [3], [15], [27]. Let $H=(G, G)$ denote the semisimple derived subgroup of $G$. The corresponding factor commutator group $G / H$ is then a torus $A$, say of dimension $s$. Since $\pi_{2}(A)$ is trivial, we obtain an exact sequence

$$
0 \longrightarrow \pi_{1}(H) \longrightarrow \pi_{1}(G) \longrightarrow \pi_{1}(A) \longrightarrow 0
$$

and thus $\pi_{1}(G) \cong \pi_{1}(H) \times \pi_{1}(A)=F \times \mathbb{Z}^{s}$, where $F=\pi_{1}(H)$ is finite. Henceforth, we shall identify $\pi_{1}(A)$ with the lattice $X_{*}(A)=\operatorname{Hom}_{\text {alg grp }}\left(\mathbb{C}^{*}, A\right)$ of co-characters (or, multiplicative one-parameter subgroups) of $A$.

To any $G$-bundle $\xi$ we may associate the torus bundle $\xi \times{ }^{G} A$ whose topological type is the image of $\gamma(\xi)$ in $\pi_{1}(A)=X_{*}(A)$. We will denote that by $c(\xi)$ and call it the Chern class of $\xi$. For any character $\chi$ in the dual lattice $X^{*}(A)=\operatorname{Hom}_{\text {alg grp }}\left(A, \mathbb{C}^{*}\right)$, the integer $\langle\chi, c(\xi)\rangle$ will then be the degree (first Chern class) of the associated line bundle $\xi \times{ }^{G} \mathbb{C}_{\chi}$.

Later, we shall have to view the Chern class $c(\xi)$ as an element of the Lie algebra $\mathfrak{g}$ of $G$. This comes about as follows. Let $C:=C(G)^{\circ}$ denote the identity component of the center of $G$. It is a torus, of dimension $s=\operatorname{dim} A$ as well, and the natural map $C \longrightarrow A$ is an isogeny of tori with kernel $C \cap H$. Thus, we may naturally identify $X_{*}(A) \otimes \mathbb{Q}$ with $X_{*}(C) \otimes \mathbb{Q}$ which, itself, may be regarded as a $\mathbb{Q}$-subspace of $\mathfrak{g}$. Viewing thus $c(\xi)$ as an element of $\mathfrak{g}$, we have the following elementary observation.

(1.1.1) Lemma. Let $\rho: G \longrightarrow G L(F)$ be a representation of $G$ on an $n$ dimensional vector space $F$, and let $d \rho: \mathfrak{g} \longrightarrow \mathfrak{g l}(F)$ be the corresponding representation of its Lie algebra. Assume that $c(\xi) \in \mathfrak{g}$ acts by a scalar, i.e. $d \rho(c(\xi))=s \cdot \operatorname{id}_{F}$ for some $s \in \mathbb{C}$. Then $s=d / n$, where $d$ is the degree of the associated vector bundle $\xi \times^{G} F$.

(1.1.2) Proof. The representation of $G$ on $\operatorname{det}(F)=\bigwedge^{n}(F)$ is given by a character $\chi$ in $X^{*}(G)=X^{*}(A)$. As remarked above, the integer $\langle\chi, c(\xi)\rangle$ equals the degree $d$ of the line bundle $\xi \times{ }^{G} \mathbb{C}_{\chi}=\xi \times{ }^{G} \operatorname{det}(F)$. But this number also agrees with the value of the infinitesimal representation of $c(\xi)$ on $\operatorname{det}(F)$, which is $n \cdot s \in \mathbb{C}=\mathfrak{g l}(\operatorname{det}(F))$. 


\section{$\S 1.2$. Stability}

Let us now turn to analytic invariants. Recall that the slope $\mu(V)$ for a holomorphic vector bundle $V$ over $E$ is defined by $\mu(V)=\operatorname{deg}(V) / \operatorname{rank}(V)$. Such a bundle is called stable (resp. semistable) if for every non-trivial holomorphic subbundle $W$ of $V$ the inequality

$$
\mu(W)<\mu(V) \quad(\text { resp. } \quad \mu(W) \leqslant \mu(V))
$$

holds. It is called unstable if it is not semistable, i.e. if there is a proper subbundle $W \subset V$ such that $\mu(W)>\mu(V)$.

The existence of a subbundle $W$ of rank $k$ inside $V$, of rank $n$, means that the structure group $G L_{n}(\mathbb{C})$ of $V$ can be reduced to a parabolic subgroup $P$ corresponding to upper diagonal block matrices with step size $k$ and $n-k$. The character group $X^{*}(P)=\operatorname{Hom}\left(P, \mathbb{C}^{*}\right)$ is freely spanned by the determinants, $\chi_{1}$ and $\chi_{2}$, on the two blocks, the global determinant det being their product, det $=\chi_{1}+\chi_{2}$ (when written additively). The characters vanishing on the center, i.e. the scalars of $G L_{n}(\mathbb{C})$ are proportional to the character $\chi=(n-$ $k) \chi_{1}-k \chi_{2}=n \chi_{1}-k$ det. Now, we have $\mu(W)<\mu(V)$ exactly when the associated line bundle $\chi(V)$ is of negative degree.

Motivated by this example, Ramanathan gave the following definition [20] for a general reductive group $G$. Call a character on a parabolic subgroup $P$ of $G$ dominant if it vanishes on the 'connected center' $C^{\circ}(G)$ of $G$ and is dominant with respect to some Borel subgroup contained in $P$.

(1.2.1) Definition. Let $G$ be a connected reductive group and $\xi$ a holomorphic principal $G$-bundle over $E$. Then $\xi$ is called stable (resp. semistable) if, for any reduction $\xi_{P}$ of $\xi$ to a parabolic subgroup $P$ of $G$ and any dominant character $\chi$ of $P$, the associated line bundle $\xi_{P} \times{ }^{P} \mathbb{C}_{\chi}$ is of negative (resp. non-positive) degree. It is called unstable if it is not semistable.

It turns out that, on an elliptic curve, there are only very few stable bundles. In particular, from the viewpoint of moduli spaces, it is reasonable to concentrate directly on the class of semistable bundles. Here are some equivalent characterizations, formulated in [9, Theorem 2.2], and based on [2], [20], [21]. We let $\mathfrak{g}$ denote the Lie algebra and $G^{a d}$ the adjoint group $G / C(G)$ of $G$. The group $G^{a d}$ is semisimple or trivial (in case $G$ is a torus).

(1.2.2) Theorem. The following conditions on a holomorphic principal Gbundle $\xi$ over $E$ are equivalent:

(a) The bundle $\xi$ is semistable. 
(b) The associated principal $G^{a d}$-bundle $\xi \times{ }^{G} G / C(G)$ (extension of structure group) is semistable.

(c) The associated adjoint vector bundle $\operatorname{ad}(\xi)=\xi \times{ }^{G} \mathfrak{g}$ is semistable.

(d) For every finite dimensional irreducible representation $\rho: G \longrightarrow$ $G L(F)$ the associated vector bundle $\xi \times^{G} F$ is semistable.

\section{§1.3. Canonical reduction}

Let $V$ be an arbitrary holomorphic vector bundle over $E$. According to a well known procedure (valid over arbitrary curves) by Harder and Narasimhan ([12], cf. e.g. [17, Ch. V]) there is a canonical filtration of $V$ by unique subbundles,

$$
0=V_{0} \subset V_{1} \subset V_{2} \subset \cdots \subset V_{r}=V
$$

such that all successive quotients $V_{i} / V_{i-1}$ are semistable of slopes $\mu_{i}=$ $\mu\left(V_{i} / V_{i-1}\right)$ with strictly decreasing values

$$
\mu_{1}>\mu_{2}>\mu_{3}>\cdots>\mu_{r}
$$

Of course, $V$ is semistable exactly when $r=1$. Since $E$ is an elliptic curve, this filtration even splits, i.e. $V$ is isomorphic to its (canonically) associated graded bundle $\operatorname{gr}(V)=\bigoplus_{i} V_{i} / V_{i-1}$. This is a standard argument using the vanishing of the higher cohomology of indecomposable vector bundels of positive degree over an elliptic curve and the additivity of the slope for tensor products of vector bundles (see e.g. [9, proof of Proposition 2.6], [2, Lemma 10.1]).

In terms of reductions, the filtration corresponds to a reduction of the structure group $G L_{n}(\mathbb{C})$ of $V$ to a parabolic subgroup $P$, and the splitting corresponds to a further reduction to a Levi subgroup $L$ of $P$.

Applying this to the adjoint bundle $\operatorname{ad}(\xi)=\xi \times{ }^{G} \mathfrak{g}$ of a given $G$-bundle $\xi$, we get a decomposition of $\operatorname{ad}(\xi)$ into a direct sum of semistable bundles $\gamma_{i}$. Let $M$ denote the additive subgroup of $\mathbb{Q}$ generated by the slopes $\mu_{i}$ of the $\gamma_{i}$ and reindex $\gamma_{i}$ as $\gamma\left(\mu_{i}\right)$. Then we may write

$$
\operatorname{ad}(\xi) \cong \bigoplus_{\nu \in M} \gamma(\nu)
$$

Invoking once more the additivity of the slope for tensor products, we easily see that $\operatorname{ad}(\xi)$ has become a bundle of $M$-graded Lie algebras. The induced grading on a special fibre, $\mathfrak{g} \cong \bigoplus_{\nu \in M} \mathfrak{g}(\nu)$, corresponds to the choice of an infinitesimal semisimple automorphism $\mu$ of the Lie algebra $\mathfrak{g}$, i.e. an element of $\operatorname{Lie}\left(G^{a d}\right)=[\mathfrak{g}, \mathfrak{g}] \subset \mathfrak{g}$. With this extra datum, the structure group $G^{a d}$ of 
$\operatorname{ad}(\xi)$ reduces to the centralizer $L^{\prime}$ of $\mu$ in $G^{a d}$, a (connected) Levi subgroup [3], $[27,6.4 .7]$. Let $L$ denote the preimage of $L^{\prime}$ in $G$. Then $L$ is a Levi subgroup of $G$ reducing the structure group of $\xi$ to $L$ (note that the bundles $\xi / L=\xi \times{ }^{G} G / L$ and $\left(\xi \times{ }^{G} G^{a d}\right) \times{ }^{G^{a d}} G^{a d} / L^{\prime}$ are the same and that respective reductions are given by sections of these bundles, cf. e.g. [11] or [21]).

As a result, the adjoint bundle $\operatorname{ad}(\xi)$ is associated to a principal $L$-bundle $\xi_{L}$ by means of the restricted adjoint representation $\operatorname{ad}_{L}: L \longrightarrow \operatorname{Aut}(\mathfrak{g})$ of $L$. Using the action of $L$ on the graded components $\mathfrak{g}(\nu)$, it may also be viewed as the direct sum of semistable associated bundles

$$
\operatorname{ad}(\xi) \cong \bigoplus_{\nu \in M} \gamma(\nu) \cong \bigoplus_{\nu \in M} \xi_{L} \otimes^{L} \mathfrak{g}(\nu)
$$

Note that $\mathfrak{g}(0)$ is the Lie algebra of $L$ and that $\xi_{L} \otimes^{L} \mathfrak{g}(0)=\operatorname{ad}\left(\xi_{L}\right)$ is a maximal semistable subbundle of subalgebras in $\xi$.

We have thus arrived, on a slightly different way, at the following basic result of Friedman and Morgan, [9, Theorem 2.7].

(1.3.1) Theorem. Let $\xi$ be an unstable holomorphic G-bundle over E. There is a proper Levi subgroup $L$ of $G$ such that

(a) $\xi$ reduces to a semistable principal $L$-bundle $\xi_{L}$,

(b) $L$ is maximal among Levi subgroups with this property.

The group $L$ is well defined up to G-conjugacy, and the bundle $\xi_{L}$ is determined up to isomorphism of principal bundles.

(1.3.2) Remark. Let $\mathfrak{p}:=\bigoplus_{\nu \in M_{\geqslant 0}} \mathfrak{g}(\nu)$. Then $\mathfrak{p}$ is the Lie algebra of a parabolic subgroup $P$ of $G$, of which $L$ is a Levi subgroup. Using the fact that the normalizer of $\mathfrak{p}$ in $G$ equals $P$ (see e.g. [27, Theorem 6.4.7]), one obtains the more familiar but weaker reduction of $\xi$ to a $P$-bundle $\xi_{P}$ already by only exploiting the inclusion $\bigoplus_{\nu \in M_{\geqslant 0}} \gamma(\nu) \subset \xi$ of Lie algebra bundles, cf. [2, Section 10] and [9, Theorem 2.7]. This procedure works over Riemann surfaces of arbitrary genus. The pair $\left(P, \xi_{P}\right)$ is usually called the canonical or HarderNarasimhan reduction of $\xi$. However, we shall stick to the following definition, special to genus 1 .

(1.3.3) Definition. The pair $\left(L, \xi_{L}\right)$, for $\xi$ unstable, (resp. $(G, \xi)$, if $\xi$ is semistable) is called the canonical reduction of $\xi$.

\section{§1.4. Atiyah-Bott type}

Since the canonically reduced bundle $\xi_{L}$ of a $G$-bundle $\xi$ is an invariant of the latter, all further invariants of $\xi_{L}$ will also be invariants of $\xi$. In particular, 
we can look at the Chern class $c\left(\xi_{L}\right)$ of $\xi_{L}$ which we may view as an element of the Lie algebra $\mathfrak{g}$ via the inclusions

$$
X_{*}(L /(L, L)) \subset \operatorname{Lie}(L) \subset \operatorname{Lie}(G)=\mathfrak{g} .
$$

In view of Theorem 1.3.1, the element $\mu(\xi):=c\left(\xi_{L}\right) \in \mathfrak{g}$ is well defined up to conjugacy by elements of $G$. Thus, we should view it as a semisimple conjugacy class in $\mathfrak{g}$ or, alternatively, as an element in the fundamental Weyl chamber $K \subset X_{*}(T) \otimes \mathbb{Q}$ for a fixed pair $(T, B)$ consisting of a maximal torus $T$ and a Borel subgroup $B \supset T$ of $G$. Because of its rôle in the work of Atiyah and Bott [2, Section 10], we define:

(1.4.1) Definition. Let $\xi$ be a $G$-principal bundle and $\xi_{L}$ its canonical reduction. Then, the Chern class $\mu(\xi)=c\left(\xi_{L}\right)$ is called the Atiyah-Bott-type of $\xi$.

(1.4.2) Remark. The semisimple element $\mu \in \mathfrak{g}$ used in the reduction process of $\xi$ is, by Lemma 1.1.1, just the image $\mu=\operatorname{ad}(\mu(\xi))$ under the adjoint representation ad $: \mathfrak{g} \longrightarrow[\mathfrak{g}, \mathfrak{g}]$. If $\xi$ is semistable, then, of course, $\mu(\xi) \in \mathfrak{c}$, the center of $\mathfrak{g}$.

(1.4.3) Remark. The Atiyah-Bott-type $\mu(\xi)$ of $\xi$ is a discrete invariant of $\xi$ since the denominators occuring in a reduced rational linear combination of elements from $X_{*}(T)$ are bounded by the exponents of the finite abelian groups $C(L)^{\circ} \cap(L, L)$.

(1.4.4) Remark. The element $\mu(\xi)$ determines the 'reducing' groups $L(\xi)$ and $P(\xi)$. Independently of the choice of a pair $(T, B)$, they may be described in the following way: $L(\xi)=C_{G}(\mu(\xi))$ and $P(\xi)=P([\mu(\xi)])$ where $P([\mu(\xi)])$ is the 'limit parabolic' attached to a one-parameter group $[\mu(\xi)]$ on the positive ray through $\mu(\xi)$, cf. [19, Ch. 2 Section 2], or [27, Proposition 8.4.5].

If we fix $\mu(\xi)$ as an element of the fundamental Weyl chamber $K \subset V:=$ $X_{*}(T) \otimes \mathbb{Q}$ associated to a pair $(T, B)$, the groups $L(\xi)$ and $P(\xi)$ will be of 'standard type'. In that respect, recall the geometric structure of $K$. Let $\Delta=\left\{\alpha_{1}, \ldots, \alpha_{\ell}\right\}$ denote a system of simple roots in $X^{*}(T)$ relative to $(T, B)$. Then $K=\{v \in V \mid \alpha(v) \geqslant 0$ for all $\alpha \in \Delta\}$ is a convex cone with faces $K_{\Theta}=\{v \in K \mid \alpha(v)=0$ for all $\alpha \in \Theta\}$ attached to arbitrary subsets $\Theta \subset \Delta$. It is the disjoint union of its relative interiors

$$
K=\dot{\bigcup}_{\Theta \subset \Delta} \dot{K}_{\Theta} \quad \text { where } \quad \dot{K}_{\Theta}=\left\{v \in K_{\Theta} \mid \alpha(v)>0 \text { for all } \alpha \in \Delta \backslash \Theta\right\} .
$$


If $\mu(\xi) \in \dot{K}_{\Theta}$, then $L(\xi)=L_{\Theta}$ and $P(\xi)=P_{\Theta}$ where $L_{\Theta}$ is generated by $T$ and the unipotent root subgroups $U_{\alpha} \alpha \in \Theta$, of $G$ and where $P_{\Theta}$ is generated by $L_{\Theta}$ and $B$. The connected center $C^{\circ}\left(L_{\Theta}\right)$ of $L_{\Theta}$ is then given by the subtorus

$$
S_{\Theta}=\{t \in T \mid \alpha(t)=1 \text { for all } \alpha \in \Theta\}^{\circ},
$$

and the vector space $X_{*}\left(S_{\Theta}\right) \otimes \mathbb{Q}=: V_{\Theta} \subset V$ is the supporting subspace for the fundamental face $K_{\Theta}$, i.e. $K_{\Theta}=V_{\Theta} \cap K$ and $\operatorname{dim} V_{\Theta}=\operatorname{dim} K_{\Theta}$.

\section{§1.5. Moduli spaces}

The canonical semistable reduction $\xi_{L}$ of a $G$-bundle $\xi$ also has continuous invariants, furnished by the corresponding point in the moduli space of semistable bundles. To these moduli spaces, we turn now.

(1.5.1) Definition. Let $\mathcal{B}$ denote a set of $G$-bundles over $E$, and let $\xi$ be a single $G$-bundle over $E$. Then $\xi$ is called adjacent to $\mathcal{B}$, or a degeneration of $\mathcal{B}$, if there is a pointed complex space $(S, s)$ and a holomorphic $G$-bundle $\Xi$ over $E \times S$ such that, for all $t \neq s$, the restricted bundle $\Xi_{t}=\left.\Xi\right|_{E \times t}$ is isomorphic to an element of $\mathcal{B}$, and $\Xi_{s}$ is isomorphic to $\xi$. A pair $((S, s), \Xi)$ as above will be called a degenerating family (from $\mathcal{B}$ to $\xi$ ).

It may happen that $\mathcal{B}$ consists of a single element $\eta$. Then we will speak of a degeneration from $\eta$ to $\xi$ or say that $\xi$ is adjacent to $\eta$. The equivalence relation generated by the adjacency relation between $G$-bundles is called $S$-equivalence.

(1.5.2) Theorem (Ramanathan). $\quad$ There exists a coarse moduli space $\mathcal{M}(G)$ of $S$-equivalence classes of semistable $G$-bundles over $E$. The connected components of $\mathcal{M}(G)$ are normal projective varieties $\mathcal{M}(G, \gamma)$ corresponding bijectively to the elements $\gamma$ in the fundamental group $\pi_{1}(G)$ of $G$ or, alternatively, to the topological isomorphism classes of $G$-bundles over $E$.

Note that, in particular, any topological $G$-bundle carries a holomorphic structure, and that the space $\mathcal{M}(G)$ is connected if $G$ is simply connected. A more explicit description is due to Friedman, Morgan, Witten and Schweigert ([10], [9, Theorems 5.19, 5.22 and 5.23], and [24], [4]). Its origin goes back to Looijenga's paper [18], which had been motivated by the deformation theory of simple elliptic singularities. Here are the most important cases.

(1.5.3) Theorem. Let $G$ be simple, simply connected and of rank $\ell$. Let $T$ be a maximal torus with Weyl group $W$ and co-character lattice $\Lambda=X_{*}(T)$. Then $\mathcal{M}(G)$ is isomorphic to the natural quotient space $\left(E \otimes_{\mathbb{Z}} \Lambda\right) / W$, a weighted projective space of dimension $\ell$. 
Now, assume that $G$ is of the form $G=\tilde{G} /\langle c\rangle$ where $\tilde{G}$ is simply connected and simple, and where $c$ is an element of the center $C(\tilde{G})$ of $\tilde{G}$. Since $\langle c\rangle$ may be identified with the fundamental group $\pi_{1}(G)$, there is a component $\mathcal{M}(G, c)$ of the moduli space $\mathcal{M}(G)$ consisting of bundles with topological type $c$. Let $\tilde{T}$ and $T=\tilde{T} / C(G)$ be corresponding maximal tori. Consider, with [5], the action of the affine Weyl groups $\hat{W}=W \ltimes X_{*}(\tilde{T})$ and $\check{W}=W \ltimes X_{*}(T)$ on $X_{*}(T) \otimes \mathbb{Q}$. The stabilizer $\check{W}_{A}$ of a fundamental alcove $A$ for the $\check{W}$-action may then be identified with the quotient $\check{W} / \hat{W} \cong X_{*}(T) / X_{*}(\tilde{T}) \cong\langle c\rangle$ as well as with a subgroup $\left\langle w_{c}\right\rangle$ of $W=\check{W} / X_{*}(T)$. This way, $\langle c\rangle$ acts as a symmetry group of $A$ and the extended Dynkin diagram of $G$ (for the explicit form, cf. the tables in [5]). Let $\ell_{c}+1$ denote the number of $\langle c\rangle$-orbits of vertices of that diagram. We shall call $\ell_{c}$ the reduced rank with respect to $c$. Then the torus $T_{c}=T /\left(\mathrm{id}-w_{c}\right) T$ is of dimension $\ell_{c}$ and acted upon by the centralizer $W_{c}=Z_{W}\left(w_{c}\right)$. Let $\Lambda_{c}$ denote its co-character lattice $X_{*}\left(T_{c}\right)$.

(1.5.4) Theorem. Let $G$ be simple of rank $\ell$ and of the form $G=\tilde{G} /\langle c\rangle$ as described above. Then $\mathcal{M}(G, c)$ is isomorphic to $\left(E \otimes_{\mathbb{Z}} \Lambda_{c}\right) / W_{c}$, a weighted projective space of dimension $\ell_{c}$.

Finally, let $G$ be reductive of the form $G=\tilde{G} /\langle c\rangle$, where $\tilde{G}=\prod_{j=0}^{r} \tilde{G}_{j}$ is a product of a central torus $\tilde{G}_{0}$ of dimension $s$ with $r$ simple and simply connected factors $\tilde{G}_{j}$, and where the element $c$ is the product of central elements $c_{j} \in \tilde{G}_{j}$. Put $G_{j}:=\tilde{G}_{j} /\left\langle c_{j}\right\rangle$.

(1.5.5) Theorem. The moduli space $\mathcal{M}(G, c)$ is an étale fiber bundle over the product $\prod_{j=1}^{r} \mathcal{M}\left(G_{j}, c_{j}\right)$ with fiber an abelian variety isogeneous to $E^{s}$.

(1.5.6) Remark. The weights occuring in the definition of the weighted projective spaces mentioned in 1.5.3 and 1.5.4 are essentially given by the coefficients of a minimal isotropic coroot (central element) of the involved affine root systems. For more details, cf. [4] as well as [16], [18], [24].

(1.5.7) Remark. One may also consider the spaces $\mathcal{M}(G, c)$ for arbitrary reductive groups $G$. They are just finite quotients of the spaces described in Theorem 1.5.5, cf. [9, Corollary 5.24].

(1.5.8) Example. Let us look at the 'classical case' studied by Atiyah [1], i.e. vector bundles of rank $n$, corresponding to principal $G L_{n}$-Bundles $\xi$ over $E$. The exact sequence

$$
1 \longrightarrow S L_{n} \longrightarrow G L_{n} \stackrel{\operatorname{det}}{\longrightarrow} \mathbb{C}^{*} \longrightarrow 1
$$


induces an isomorphism $\pi_{1}\left(G L_{n}\right) \cong \mathbb{Z}$ which identifies the topological type of $\xi$ with the degree of the associated vector bundle $\xi \times{ }^{G L_{n}} \mathbb{C}^{n}$. In the case that the determinant bundle $\xi \times^{G L_{n}} \bigwedge^{n} \mathbb{C}^{n}$ is trivial, the group of the bundle reduces to $S L_{n}$ and, according to Theorem 1.5.3 and the respective references, we have

$$
\mathcal{M}\left(G L_{n}, 0\right)=\mathcal{M}\left(S L_{n}\right) \cong\left(E^{n-1}\right) / S_{n} \cong \mathbb{P}^{n-1}(\mathbb{C}) .
$$

Next, consider bundles with structure group $P G L_{n}=S L_{n} / \mu_{n}$. Then

$$
\pi_{1}\left(P G L_{n}\right)=\mu_{n}=\left\{e^{2 \pi i m / n} \mid m=0,1, \ldots, n-1\right\} .
$$

Let $c=e^{2 \pi i m / n} \in \mu_{n}=C\left(S L_{n}\right)$ and $g=\operatorname{gcd}(m, n)$. The extended Dynkin diagram $\tilde{\Delta}$ may be identified with the unit circle $S^{1} \subset \mathbb{C}$, the nodes being given by the subset $\mu_{n}$. Then $c$ acts simply by multiplication, i.e. through rotation by $2 \pi m / n$, yielding $g$ orbits of nodes for the group $\langle c\rangle$. According to Theorem 1.5 .4 , resp. the relevant references, we now have

$$
\mathcal{M}\left(P G L_{n}, c\right) \cong\left(E^{g-1}\right) / S_{g} \cong \mathbb{P}^{g-1}(\mathbb{C}) .
$$

Finally, consider $G L_{n}$-bundles $\xi$ of arbitrary degree $m \in \mathbb{Z}$, and put $g=$ $\operatorname{gcd}(m, n)$ again. From the sequence

$$
1 \longrightarrow \mathbb{C}^{*} \longrightarrow G L_{n} \longrightarrow P G L_{n} \longrightarrow 1,
$$

and the 'anti-diagonal' quotient realisation $G L_{n}=\left(\mathbb{C}^{*} \times S L_{n}\right) / \mu_{n}$ we see that the topological type of the extended $P G L_{n}$-bundle $\bar{\xi}=\xi \times{ }^{G L_{n}} P G L_{n}$ is $c=$ $e^{-2 \pi i m / n}$. Thus, by Theorem 1.5.5, we have a fibering

$$
\mathcal{M}\left(G L_{n}, m\right) \longrightarrow \mathcal{M}\left(P G L_{n}, e^{-2 \pi i m / n}\right) \cong \mathbb{P}^{g-1}(\mathbb{C}),
$$

whose fibre may, in fact, be identified with $E /{ }_{f} E \cong E$ (here $n=f . g$, and ${ }_{f} E$ denotes the $f$-torsion points of $E$ ).

\section{§2. Automorphism Groups}

Our final interest will be in the orbital geometry of 'big' or 'almost generic' bundles. These are principal $G$-bundles over $E$ which, in any of their local deformations $((S, s), \Xi)$ with $\Xi_{s} \cong \xi$, are found over a space of small codimension in $S$. This corresponds to a small infinitesimal deformation space $H^{1}(E, \operatorname{ad}(\xi))$, or, using Serre and Killing duality, to a small automorphism group, $\operatorname{Lie}(\operatorname{Aut}(\xi)) \cong H^{0}(E, \operatorname{ad}(\xi))$. We shall therefore investigate the automorphism groups of $G$-bundles in this section. 
As is easily seen, there are unstable bundles with automorphism groups of arbitrarily large dimension. In contrast, as shown below, 2.2, all semistable bundles have an automorphism group embeddable into the original group $G$. In that sense, they all may be considered to be big.

(2.1) Definition. Let $G$ be reductive. A semistable $G$-bundle is called regular (resp. semisimple) if its automorphism group has minimal dimension among all semistable $G$-bundles of the same topological type (resp. maximal dimension among all members in its semistable $S$-equivalence class).

For simple groups $G=\tilde{G} /\langle c\rangle$ of the type considered in Theorems 1.5.3 and 1.5.4, we have the following result which is a reformulation, from the angle of $[26,3.10]$, of results and constructions contained in [9, especially Theorem 4.1, Corollaries 6.2 and 6.3].

(2.2) Theorem. Each S-equivalence class [ $[\xi]$ of semistable bundles in $\mathcal{M}(G, c)$ consists of finitely many isomorphism classes which are in a bijective, adjacency-preserving correspondence with the the unipotent conjugacy classes in a reductive subgroup $Z([\xi])$ of rank $\ell_{c}$ in $G$. Under this correspondence, the automorphism group $\operatorname{Aut}_{G}(\xi)$ is identified with the stabilizer of the corresponding unipotent element in $Z([\xi])$. Thus, for all $\xi \in[\xi]$ we have

$$
\ell_{c} \leqslant \operatorname{dim}_{A^{\prime}}(\xi) \leqslant \operatorname{dim} Z([\xi]) \text { and } \operatorname{dim}_{G} \operatorname{Aut}_{G}(\xi) \equiv \ell_{c} \quad(\bmod 2) .
$$

There are unique isomorphism classes of regular and of semisimple bundles in $[\xi]$. If $\xi$ is a regular bundle, then $\operatorname{Aut}_{G}(\xi)^{\circ}$ is an abelian subgroup of $Z([\xi])$ of dimension $\ell_{c}$. If $\xi$ is a semisimple bundle, then $\operatorname{Aut}_{G}(\xi)=Z([\xi])$. Furthermore, there is an open dense subset $\mathcal{M}(G, c)_{\text {rs }} \subset \mathcal{M}(G, c)$ whose members $[\xi]$ consist of a single, regular and semisimple, isomorphism class.

Call a semistable $G$-bundle $\xi$ with $[\xi] \in \mathcal{M}(G, c)$, subregular if $\operatorname{dim}^{\operatorname{Aut}_{G}(\xi)}$ $=\ell+2$. Then we can add the following to $(2.2)$, using $[26,5.4]$.

(2.3) Addition. An $S$-equivalence class $[\xi]$ of semistable bundles in $\mathcal{M}(G, c)$ contains subregular bundles if and only if $Z([\xi])^{\circ}$ is not a torus, i.e. of dimension $\geqslant \ell+2$. In that case, the isomorphism classes of subregular bundles are in bijection with the subregular unipotent classes in $Z([\xi])$ which are, themselves, in bijection with the orbits of the action of the component group $Z([\xi]) / Z([\xi])^{\circ}$ on the simple (quasi-) factors of $Z([\xi])^{\circ}$.

Let now $\xi$ be an unstable $G$-bundle. We can get useful insight into its automorphism group $\operatorname{Aut}_{G}(\xi)$ by exploiting its canonical reduction $\left(L, \xi_{L}\right)$ and 
its Atiyah-Bott type $\mu(\xi)$. Let $P=P(\xi) \supset L$ be the associated parabolic. Recall that the Lie algebra of $\operatorname{Aut}_{G}(\xi)$ can be written as the space of global sections of the adjoint bundle. Using the decomposition $\mathfrak{g}=\bigoplus_{\nu \in M} \mathfrak{g}(\nu)$ of $\mathfrak{g}$ with respect to $\mu(\xi)$, we get

$$
\begin{aligned}
\operatorname{Lie}\left(\operatorname{Aut}_{G}(\xi)\right) & =H^{0}(E, \operatorname{ad}(\xi))=H^{0}\left(E, \xi_{L} \times^{L} \mathfrak{g}\right) \\
& =H^{0}\left(E, \operatorname{ad}\left(\xi_{L}\right)\right) \oplus \bigoplus_{\nu \in M_{>0}} H^{0}\left(E, \xi_{L} \times{ }^{L} \mathfrak{g}(\nu)\right)=H^{0}\left(E, \operatorname{ad}\left(\xi_{P}\right)\right)
\end{aligned}
$$

(since only non-negative degrees contribute). The dimension can be computed in an elegant way. Namely, $\mathfrak{g}(\nu)$ is spanned by the root spaces $\mathfrak{g}_{\alpha}$ for all roots $\alpha$ with $\alpha(\mu(\xi))=\nu$ (and $\mathfrak{h}$, if $\nu$ is zero). Since $\nu$ is the slope of $\xi_{L} \times{ }^{L} \mathfrak{g}(\nu)$, the degree of $\xi_{L} \times{ }^{L} \mathfrak{g}(\nu)$, and hence the dimension of $H^{0}\left(E, \xi_{L} \times{ }^{L} \mathfrak{g}(\nu)\right)$ with $\nu>0$, is given by $\nu \cdot \operatorname{dim} \mathfrak{g}(\nu)$. Thus

$$
\operatorname{dim}\left(\bigoplus_{\nu \in M_{>0}} H^{0}\left(E, \xi_{L} \otimes^{L} \mathfrak{g}(\nu)\right)\right)=\sum_{\alpha \in \Sigma^{+}} \alpha(\mu(\xi)) .
$$

As usual ([5]), denote the half sum of the positive roots by $\rho \in X^{*}(T) \otimes$ $\mathbb{Q}$, and let $\operatorname{Aut}_{G}(\xi)^{+}$be the connected subgroup of $\operatorname{Aut}_{G}(\xi)$ corresponding to the subalgebra $\bigoplus_{\nu \in M_{>0}} H^{0}\left(E, \xi_{L} \times{ }^{L} \mathfrak{g}(\nu)\right)$ of Lie $\left(\operatorname{Aut}_{G}(\xi)\right)$. Collecting the above information, we obtain the following statement (cf. also $[2,10.7]$ and $[9$, Theorem 2.7]).

(2.4) Proposition. Let $\xi$ be an unstable $G$-bundle, $\left(L, \xi_{L}\right)$ its canonical reduction and $\mu(\xi)$ its Atiyah-Bott type. Let $P=P(\xi) \supset L$ be the associated parabolic.

(a) We have equality for the identity components, $\operatorname{Aut}_{G}(\xi)^{\circ}=\operatorname{Aut}_{P}\left(\xi_{P}\right)^{\circ}$.

(b) The group $\operatorname{Aut}_{G}(\xi)^{+}$is a unipotent normal subgroup of $\operatorname{Aut}_{G}(\xi)$ of dimension $\langle 2 \rho, \mu(\xi)\rangle$, and $\operatorname{Aut}_{G}(\xi)^{\circ}$ is a semidirect product $\operatorname{Aut}_{L}\left(\xi_{L}\right)^{\circ} \ltimes$ $\operatorname{Aut}_{G}(\xi)^{+}$.

(c) $\operatorname{dim}_{\operatorname{Aut}_{G}}(\xi)=\operatorname{dim}_{\mathrm{Aut}_{L}}\left(\xi_{L}\right)+\langle 2 \rho, \mu(\xi)\rangle$.

According to this result, the problem of determining the dimension of $\operatorname{Aut}_{G}(\xi)$ is now broken into

(i) the computation of $\operatorname{dim} \operatorname{Aut}_{L}\left(\xi_{L}\right)$, and

(ii) the evaluation of $\langle 2 \rho, \mu(\xi)\rangle$.

Concerning (i), we have Theorem 2.2 which, however, has to be completed by a precise description of the structure of the Levi subgroups of $G$. Concerning (ii), we have to determine the possible Atiyah-Bott types $\mu(\xi)$. In the following, we will address both problems in the simplest cases, i.e. for those bundles $\xi$ whose parabolic type is a maximal parabolic subgroup $P$ of $G$. 
Let us fix some notations. Following [5], a system of simple roots in $X^{*}(T)$ will be denoted by $\Delta=\left\{\alpha_{1}, \ldots, \alpha_{\ell}\right\}$. Then there are well-defined fundamental co-weights $\varpi_{k}^{\vee} \in X_{*}(T \cap(G, G)) \otimes \mathbb{Q} k=1, \ldots, \ell$, i.e. $\left\langle\alpha_{i}, \varpi_{k}^{\vee}\right\rangle=\delta_{i k}$ with respect to the natural pairing. If $G$ is semisimple, we also fix fundamental dominant weights $\varpi_{k} \in X^{*}(T) \otimes_{Z} \mathbb{Q}$. Let $\Lambda$ be any integer lattice and $\omega \in \Lambda \otimes \mathbb{Q}$. We shall write $[\omega]$ for the smallest positive integer multiple of $\omega$ which lies in $\Lambda$.

(2.5) Definition. Let $\xi$ be an unstable $G$-bundle with associated parabolic subgroup $P \subset G$ and topological type $\gamma \in \pi_{1}(G)$. The bundle $\xi$ is called $P$ maximal if its automorphism group $\operatorname{Aut}_{G}(\xi)$ has minimal dimension among all bundles with associated parabolic $P$. It is called $\gamma$ - $P$-maximal if its automorphism group has minimal dimension among all bundles of topological type $\gamma$ with associated parabolic $P$.

In the following, we shall view $\mu(\xi)$ as an element of a fixed fundamental chamber. The associated parabolics are then 'standard'. We put $P_{k}:=P_{\Delta \backslash\left\{\alpha_{k}\right\}}$ for the maximal standard parabolic subgroup of $G$ attached to a simple root $\alpha_{k} \in \Delta$.

(2.6) Theorem. $\quad$ Let $G$ be semisimple and $\xi$ an unstable $G$-bundle of type $P_{k}$. If $\xi$ is $P_{k}$-maximal, we have

$$
\begin{aligned}
\mu(\xi) & =\varpi_{k}^{\vee} /\left\langle\left[\varpi_{k}\right], \varpi_{k}^{\vee}\right\rangle, \quad \text { i.e. } \\
\operatorname{dim} \operatorname{Aut}_{G}(\xi)^{+} & =\left\langle 2 \rho, \varpi_{k}^{\vee}\right\rangle /\left\langle\left[\varpi_{k}\right], \varpi_{k}^{\vee}\right\rangle .
\end{aligned}
$$

If $\xi$ is not necessarily $P_{k}$-maximal, its Atiyah-Bott type is a positive integral multiple of the element $\varpi_{k}^{\vee} /\left\langle\left[\varpi_{k}\right], \varpi_{k}^{\vee}\right\rangle$.

(2.7) Proof. Put $P=P_{k}, L=L_{\Delta \backslash\left\{\alpha_{k}\right\}}$, and $S=S_{\Delta \backslash\left\{\alpha_{k}\right\}}$. Consider the composition of group homomorphisms

$$
\phi: \mathbb{C}^{*} \cong S \longrightarrow L \longrightarrow A=L /(L, L)=P /(P, P) \cong \mathbb{C}^{*} .
$$

On the level of co-characters, the map $\phi_{*}: \mathbb{Z}=X_{*}(S) \longrightarrow X_{*}(A)=\mathbb{Z}$ is given by multiplication with the degree $\operatorname{deg}(\phi)$. Note that $X_{*}(S)=\mathbb{Z}\left[\varpi_{k}^{\vee}\right]$, and that $\phi$ is given by the character $\left[\varpi_{k}\right]$ restricted to $S \subset T$. Thus, $\operatorname{deg}(\phi)=\left\langle\left[\varpi_{k}\right],\left[\varpi_{k}^{\vee}\right]\right\rangle$, and $X_{*}(A)$ may be identified with the sublattice $\mathbb{Z}\left[\varpi_{k}^{\vee}\right] /\left\langle\left[\varpi_{k}\right],\left[\varpi_{k}^{\vee}\right]\right\rangle \subset X_{*}(S) \otimes$ $\mathbb{Q}$. A minimal Chern class $\mu(\xi) \in X_{*}(T) \otimes \mathbb{Q}$ for a bundle $\xi$ with associated parabolic $P_{k}$ is thus given by

$$
\left[\varpi_{k}^{\vee}\right] /\left\langle\left[\varpi_{k}\right],\left[\varpi_{k}^{\vee}\right]\right\rangle=\varpi_{k}^{\vee} /\left\langle\left[\varpi_{k}\right], \varpi_{k}^{\vee}\right\rangle
$$


(2.8) Example. Let us look at simple groups of type $A_{\ell}$, i.e. $G=S L_{n} / \mu_{g}$, where $g$ is a divisor of $n=\ell+1$. Then, using the tables of [5] and their notation, one can show

$$
\begin{gathered}
{\left[\varpi_{k}\right]=\kappa \cdot \varpi_{k} \quad \text { with } \quad \kappa=g /(g, k) \quad \text { and thus }} \\
\left\langle 2 \rho, \varpi_{k}^{\vee}\right\rangle /\left\langle\left[\varpi_{k}\right], \varpi_{k}^{\vee}\right\rangle=(g, k) \cdot(n / g) .
\end{gathered}
$$

Here $(a, b)$ denotes the greatest common divisor of the integers $a$ and $b$. In particular, for $S L_{n},(g=1)$, the simply connected case, we get the value $n$ for all $k$, and for $P G L_{n}$ (where $\left.g=n\right)$, we get $\left\langle 2 \rho, \varpi_{k}^{\vee}\right\rangle /\left\langle\left[\varpi_{k}\right], \varpi_{k}^{\vee}\right\rangle=(n, k)$.

Due to the complications, related to the embedding of the lattices $X_{*}(S) \subset$ $X_{*}(A)$ and their intersection with the fundamental Weyl chamber, it seems practically impossible, apart from individual cases, to develop similar formulae or, at least, reasonable estimates for arbitrary parabolic subgroups. For our purposes, some general geometric properties will do. They may also be of independent interest.

\section{§3. Degenerations}

Let $G$ be reductive, again, with pair $(\mathrm{T}, \mathrm{B})$ fixing a fundamental Weyl chamber $K$ in $X_{*}(T) \otimes \mathbb{Q}$. On $X_{*}(T) \otimes \mathbb{Q}$ we fix some invariant scalar product, which we shall also denote by $\langle$,$\rangle , thus identifying X_{*}(T) \otimes \mathbb{Q}$ with its dual $X^{*}(T) \otimes \mathbb{Q}$. Note that the central part $X_{*}(C(G)) \otimes \mathbb{Q}$ will split off as an orthogonal summand. Let $L=L_{\Theta}$ be a standard Levi subgroup with connected center $S \subset T$ and commutative quotient $A=L /(L, L)$.

(3.1) Lemma. Under the identification of $X_{*}(A) \otimes \mathbb{Q}$ with $X_{*}(S) \otimes \mathbb{Q}$ induced by the isogeny $S \longrightarrow A$, the projection $\pi: X_{*}(T) \otimes \mathbb{Q} \longrightarrow X_{*}(A) \otimes \mathbb{Q}$ coincides with the orthogonal projection along the subspace $X_{*}(T \cap(L, L)) \otimes \mathbb{Q}$.

(3.2) Proof. Since $X_{*}(T \cap(L, L)) \otimes \mathbb{Q}$ is the kernel of $\pi$, we only have to show that this space is orthogonal to $X_{*}(S) \otimes \mathbb{Q}$. But these spaces are generated by the simple co-roots $\alpha^{\vee}, \alpha \in \theta$, resp. the fundamental co-weights $\varpi_{\beta}^{\vee}$ with $\beta \in \Delta \backslash \Theta$ and the center $X_{*}(C(G)) \otimes \mathbb{Q}$, which are orthogonal to each other.

Recall the notion of adjacency, 1.5.1.

(3.3) Proposition. $\quad$ Let $\xi$ be an unstable $G$-bundle of Atiyah-Bott type $\mu(\xi)$ lying in $X_{*}(T /(T \cap(P, P)))$ with associated parabolic $P$. Let $Q$ be a parabolic 
containing $P$. Then $\xi$ is adjacent to some unstable bundle of Atiyah-Bott type $\pi(\mu(\xi))$ lying in $X_{*}(T /(T \cap(Q, Q)))$ with associated parabolic $Q$. In particular, any unstable $G$-bundle is adjacent to a semistable one.

(3.4) Proof. Let us first reduce the general case to the special one $(Q=G)$. From the canonical reduction $\left(L, \xi_{L}\right)$ of $\xi$ to the (standard) Levi subgroup $L \subset P$ we obtain another, intermediate reduction $\xi_{M}=\xi_{L} \times{ }^{L} M$ of $\xi$ by extension to the (standard) Levi subgroup $M \supset L$ of $Q$. By the construction of the canonical reduction, $\xi_{M}$ will be unstable and, granting the special case of our claim for the group $M$, there will be a degenerating family $\left((S, s), \Xi_{M}\right)$ from a semistable $M$-bundle $\eta_{M}$ to $\xi_{M}$. This family extends to a family of $G$-bundles $((S, s), \Xi)$, where $\Xi=\Xi_{M} \times{ }^{M} G$, degenerating $\eta=\eta_{M} \times{ }^{M} G$ into $\xi$. We now have to determine the Atiyah-Bott type $\mu(\eta)$ of $\eta$.

Note that the natural projection $\pi: X_{*}\left(T /(T \cap(L, L)) \longrightarrow X_{*}(T /(T \cap\right.$ $(M, M))$ maps the Chern class $c\left(\xi_{L}\right)(=\mu(\xi))$ of $\xi_{L}$ to $c\left(\xi_{M}\right)$. Since Chern classes are topological invariants, they do not change in flat families, thus $c\left(\eta_{M}\right)=c\left(\xi_{M}\right)$. Using Lemma 3.1 and the acuteness of the dihedral angles of the fundamental Weyl chamber $K$ (i.e. $\langle\alpha, \beta\rangle \leqslant 0$ for all $\alpha \neq \beta$ in $\Delta$ ), we see that $\pi$ maps the interior of the face $K \cap X_{*}(T /(T \cap(L, L)) \otimes \mathbb{Q}$ to the interior of the face $K \cap X_{*}(T /(T \cap(M, M)) \otimes \mathbb{Q}$. Thus, the element $\pi(\mu(\xi))$ coincides with the Atiyah-Bott type $\mu(\eta)$.

We finally have to prove the special case $(Q=G)$. Here, we need some insights from the deformation theory of $\xi$, which should be well-known. They are consequences of the parametrization of $G$-bundles in terms of $(0,1)$-connections, [2], or of loop groups (for some facts, cf. [2, Section 15]). Because of $\operatorname{dim}_{\mathbb{C}}(E)=$ 1 , the deformation theory of $\xi$ is unobstructed due to $H^{2}(E, \operatorname{ad}(\xi))=0$. We thus have a semi-universal deformation $((\mathfrak{U}, 0), \Xi)$ of $\xi$ with smooth base space $(\mathfrak{U}, 0)$,

$$
T_{0} \mathfrak{U}=H^{1}(E, \operatorname{ad}(\xi))=H^{0}(E, \operatorname{ad}(\xi))^{\vee},
$$

on which the automorphism group $\operatorname{Aut}_{G}(\xi)$ acts. There is then a multiplicative subgroup $M \cong \mathbb{C}^{*} \subset \operatorname{Aut}_{L}\left(\xi_{L}\right)^{\circ} \subset \operatorname{Aut}_{G}(\xi)$ given by an integer multiple of $\mu(\xi)$ inside $X_{*}(T)$ which acts on $\mathfrak{U}$ with non-positive weights. Let $\mathfrak{U}_{-}$be the smooth subspace of $\mathfrak{U}$ which is contracted to the base point 0 by the action of $M$ (when going to $\infty$ ). The bundle $\Xi_{u}$ for a generic $u \in \mathfrak{U}_{-}$will be semistable. Now, let $S$ be the closure of the $M$-orbit of $u$ inside $\mathfrak{U}_{-}$. The restricted family $\left((S, 0),\left.\Xi\right|_{S}\right)$ is a degeneration as requested.

There is a much more general result first proved for vector bundles by Shatz [25] and then reformulated and generalized to arbitrary structure groups 
by Atiyah and Bott $[2,7.8,10.6,12.8]$ which, in particular, gives a necessary criterion for adjacency. In the following, the Atiyah-Bott type $\mu(\xi)$ of a $G$ bundle $\xi$ will be considered as an element in $V=X_{*}(T) \otimes \mathbb{R}$, and we will denote by $\widehat{W \mu}$ the convex hull of the Weyl group orbit of an element $\mu$ in $V$.

(3.5) Theorem (Atiyah-Bott). Let $\Xi$ be a family of $G$-bundles over the pointed space $(S, s)$, i.e. a holomorphic $G$-bundle over the product space $E \times S$. Assume that for all $t \neq s$ the Atiyah-Bott type $\mu\left(\Xi_{t}\right)$ is a constant $\mu$, and let $\Xi_{s}$ be of Atiyah-Bott type $\mu_{0}$. Then $\mu$ is contained in the convex hull $\widehat{W \mu_{0}}$ of the $W$-orbit of $\mu_{0}$.

The convex hull of $W \mu_{0}$ can be conveniently described, cf. [2, Lemma 12.14].

(3.6) Lemma. Let $\mu$ and $\mu_{0}$ be elements of the fundamental chamber $C \subset V$. Then $\mu \in \widehat{W \mu_{0}} \Longleftrightarrow$ for all $\omega \in C$ we have $\langle\omega, \mu\rangle \leqslant\left\langle\omega, \mu_{0}\right\rangle$.

(3.7) Example. In Proposition (3.3), we have a degeneration of Atiyah-Bott type $\mu=\pi\left(\mu_{0}\right)$ into Atiyah-Bott type $\mu_{0}=\mu(\xi)$, where $\pi$ is the orthogonal projection onto a face $C^{\prime}$ of $C$. A quick way to see that $\mu \in \widehat{W \mu_{0}}$ is to write $\mu$ as a weighted sum over the stabilizer $W^{\prime}$ of $C^{\prime}$ in $W$ :

$$
\mu=\pi\left(\mu_{0}\right)=\frac{1}{\left|W^{\prime}\right|} \sum_{w \in W^{\prime}} w\left(\mu_{0}\right) .
$$

(3.8) Example. Let $G=\operatorname{Spin}_{10}$ of type $D_{5}$. For any $k=1, \ldots, 5$, there are $P_{k}$-maximal unstable $G$-bundles of Atiyah-Bott type $\mu_{k}=\varpi_{k} /\left\langle\varpi_{k}, \varpi_{k}\right\rangle$ (we are identifying weights and co-weights). In a later context, we are interested in the question whether there exist families of $G$-bundles, whose generic member is of type $\mu_{2}$ and whose special member has type $\mu_{5}$. For that, we check the inequality of Lemma 3.6 on the element $\omega=2 \mu_{2} \in C$. In the notations of [5], we have:

$$
\begin{array}{ll}
\mu_{2}=\left(\varepsilon_{1}+\varepsilon_{2}\right) / 2, & \mu_{5}=2\left(\varepsilon_{1}+\varepsilon_{2}+\varepsilon_{3}+\varepsilon_{4}+\varepsilon_{5}\right) / 5, \\
\left\langle\omega, \mu_{2}\right\rangle=1 \quad \text { and } & \left\langle\omega, \mu_{5}\right\rangle=4 / 5 .
\end{array}
$$

Thus, there are no such families.

The following rounds off our geometric discussion.

(3.9) Proposition. Let $\xi$ and $\eta$ be G-bundles. If $\xi$ is adjacent to $\eta$, then

$$
\operatorname{dim} \operatorname{Aut}(\xi)>\operatorname{dim} \operatorname{Aut}(\eta) .
$$


(3.10) Proof. Let $((S, s), \Xi)$ be a degeneration of $\eta$ into $\xi$. Locally at $s$, this family is induced by a morphism $\phi$ from the germ $(S, s)$ to the base space $\mathfrak{U}$ of the semi-universal deformation of $\xi$. Because of the non-triviality of the family $\Xi$, the image of $\phi$ has to have positive dimension. Openness of versality inside $\mathfrak{U}$ now implies $\operatorname{dim} H^{1}(E, \operatorname{ad}(\eta))<\operatorname{dim} H^{1}(E, \operatorname{ad}(\xi))$. Serre and Killing duality give the corresponding inequality for the Lie algebras of the automorphism groups.

\section{$\S 4$. On the Structure of Levi Subgroups}

We will now turn to the structure of the maximal Levi subgroups of $G$. Let us start with the following general recollections.

(4.1) Lemma. Let $G$ be semisimple and $L \subset G$ a Levi subgroup $L=L_{\Theta} \subset$ $P_{\Theta}$ associated with a subset $\Theta \subset \Delta$. Denote the semisimple derived subgroup $(L, L)$ of $L$ by $H$ and put $S=C(L)^{\circ}$.

(a) The group $L$ is an almost direct product of $H$ and $S$, i.e. $L=S . H$ and $S \cap H$ is finite. $H$ itself is an almost direct product $H=H_{1} H_{2} \cdots H_{r}$ of simple groups $H_{j}$ corresponding to the connected components of $\Theta$.

(b) If $L$ is maximal, i.e. $L=L_{k}=L_{\Theta}$ where $\Theta=\Delta \backslash\left\{\alpha_{k}\right\}$, then $S \cap H$ is a cyclic subgroup of $S \cong \mathbb{C}^{*}$ of order $d=\left\langle\left[\varpi_{k}\right],\left[\varpi_{k}^{\vee}\right]\right\rangle$.

(c) If $G$ is simply connected, the groups $H, H_{j}$ are as well, and $H$ is a direct product $H=\prod_{j=1}^{r} H_{j}$.

(4.2) Proof. These facts are well known (cf. e.g. [27, Chap. 8, esp. 8.4.6. (6)]). If $L$ is maximal, we only have to add that the subtorus $S \cong \mathbb{C}^{*}$ is generated by $\left[\varpi_{k}^{\vee}\right] \in X_{*}(T)$, and that the intersection $S \cap H$ equals the kernel of the character $\left[\varpi_{k}\right]: L \longrightarrow \mathbb{C}^{*}$, restricted to $S$.

The following lemma provides some topological information.

(4.3) Lemma. Let $G$ be semisimple and $L \subset G$ a Levi subgroup $L=L_{\Theta} \subset$ $P_{\Theta}$ associated with a subset $\Theta \subset \Delta$. Denote the semisimple derived subgroup $(L, L)$ of $L$ by $H$ and the connected centre $C(L)^{\circ}$ by $S$. Put $\bar{S}=L / H=S / S \cap H$ and $\bar{H}=L / S=H / S \cap H$. Then the long exact homotopy sequences associated with the fibrations

$$
\begin{aligned}
& H \longrightarrow L \longrightarrow L / H, \quad S \longrightarrow L \longrightarrow L / S, \\
& L \longrightarrow G \longrightarrow G / L, \quad H \longrightarrow G \longrightarrow G / H
\end{aligned}
$$


induce short exact sequences

$$
\begin{aligned}
& 0 \longrightarrow \pi_{1}(H) \longrightarrow \pi_{1}(L) \longrightarrow \pi_{1}(\bar{S}) \longrightarrow 0 \\
& 0 \longrightarrow \pi_{1}(S) \longrightarrow \pi_{1}(L) \longrightarrow \pi_{1}(\bar{H}) \longrightarrow 0 \\
& 0 \longrightarrow \pi_{2}(G / L) \longrightarrow \pi_{1}(L) \longrightarrow \pi_{1}(G) \longrightarrow 0 \\
& 0 \longrightarrow \pi_{1}(H) \longrightarrow \pi_{1}(G) \longrightarrow \pi_{1}(G / H) \longrightarrow 0
\end{aligned}
$$

(4.4) Proof. These sequences are easy consequences of the vanishing $\pi_{2}(\bar{S})=$ $0, \pi_{2}(\bar{H})=0, \pi_{1}(G / L)=\pi_{1}(G / P)=0, \pi_{2}(G)=0$ and $\pi_{2}(G / H)=0$. For the last equality, observe that $G / H$ is finitely covered by $\tilde{G} / \tilde{H}$, where $\tilde{G}$ and $\tilde{H}$ are the simply connected coverings of $G$ and $H$, and that $\pi_{2}(\tilde{G} / \tilde{H})=0$ (use $\pi_{1}(\tilde{H})=\pi_{1}(\tilde{G})=0$, Lemma 4.1).

(4.5) Remark. Henceforth, we shall call the map $\pi_{1}(L) \longrightarrow \pi_{1}(\bar{S})$ from the first sequence the degree map. If $L=L_{k}$ is maximal and $\eta$ an $L$-bundle of topological type $\gamma=\gamma(\eta)$ in $\pi_{1}(L)$, then the image of $\gamma$ in $\pi_{1}(\bar{S})=\mathbb{Z}$ is an integer, the degree of the line bundle $\eta \times{ }^{L} \mathbb{C}$ associated with the character $\left[\varpi_{k}\right]$.

(4.6) Remark. For computational questions, especially like those to come in the non-simply connected case, it may be helpful to interpret the exact sequences of Lemma 4.3 in terms of lattices related to $X_{*}(T)=\pi_{1}(T)$. For any simple root $\alpha$, let $\alpha^{\vee} \in X_{*}(T)$ denote the corresponding co-root and $\varpi_{\alpha} \in$ $X^{*}(T) \otimes \mathbb{Q}$ the corresponding fundamental dominant weight. Let $T^{\prime}$ be the subtorus $T \cap H$ and $\bar{T}$ the quotient torus $T / S=T \cap H / S \cap H$. We have

$$
\begin{aligned}
X_{*}(S) & =\left\{\gamma \in X_{*}(T) \mid\langle\alpha, \gamma\rangle=0 \text { for all } \alpha \in \Theta\right\} \quad \text { and } \\
X_{*}\left(T^{\prime}\right) & =\left\{\gamma \in X_{*}(T) \mid\left\langle\varpi_{\beta}, \gamma\right\rangle=0 \text { for all } \beta \in \Delta \backslash \Theta\right\} .
\end{aligned}
$$

Let $Q^{\vee}$, resp. $Q^{\prime \vee}$, denote the sublattice of $X_{*}(T)$ generated by all dual roots, resp. by those dual to $\Theta$. Then the above four sequences can be written in the following form

$$
\begin{aligned}
& 0 \longrightarrow X_{*}\left(T^{\prime}\right) / Q^{\prime \vee} \longrightarrow X_{*}(T) / Q^{\prime \vee} \longrightarrow \quad X_{*}(T) / X_{*}\left(T^{\prime}\right) \quad \longrightarrow 0 \\
& 0 \longrightarrow X_{*}(S) \quad \longrightarrow X_{*}(T) / Q^{\prime \vee} \longrightarrow \quad X_{*}(\bar{T}) / Q^{\prime \vee} \quad \longrightarrow 0 \\
& 0 \longrightarrow Q^{\vee} / Q^{\prime \vee} \longrightarrow X_{*}(T) / Q^{\prime \vee} \longrightarrow \quad X_{*}(T) / Q^{\vee} \quad \longrightarrow 0 \\
& 0 \longrightarrow X_{*}\left(T^{\prime}\right) / Q^{\prime \vee} \longrightarrow X_{*}(T) / Q^{\vee} \longrightarrow X_{*}(T) /\left(X_{*}\left(T^{\prime}\right)+Q^{\vee}\right) \longrightarrow 0
\end{aligned}
$$

In the third sequence, we have the interpretation

$$
\pi_{2}(G / L)=\pi_{2}(G / P)=H_{2}(G / P, \mathbb{Z})=Q^{\vee} / Q^{\prime \vee}=\bigoplus_{\beta \in \Delta \backslash \Theta} \mathbb{Z} \beta^{\vee}
$$


(4.7) Remark. The first two of the sequences in 4.3, together with the covering group sequences,

$$
\begin{aligned}
& 0 \longrightarrow \pi_{1}(S) \longrightarrow \pi_{1}(\bar{S}) \longrightarrow \pi_{0}(S \cap H)=S \cap H \longrightarrow 0 \\
& 0 \longrightarrow \pi_{1}(L) \longrightarrow \pi_{1}(\bar{H}) \longrightarrow \pi_{0}(S \cap H)=S \cap H \longrightarrow 0
\end{aligned}
$$

give rise to an exact sequence of the following form.

$$
0 \longrightarrow \pi_{1}(L) \longrightarrow \pi_{1}(\bar{S}) \times \pi_{1}(\bar{H}) \stackrel{+}{\longrightarrow} \pi_{0}(S \cap H)=S \cap H \longrightarrow 0,
$$

which embeds $\pi_{1}(L)$ into the product $\pi_{1}(\bar{S}) \times \pi_{1}(\bar{H})$ as the subset of all pairs $(\alpha, \beta)$ such that $\pi(\alpha)=-\pi(\beta)$. Here $\pi$ denotes the respective projection to $S \cap H$. This is a consequence of general covering theoretic facts, cf. also $[2$, Ch. 6].

Here is a result in the simply connected case.

(4.8) Corollary. Assume that $G$ is simply connected and that $L$ is a maximal Levi subgroup $L=L_{k}$. Let $d=\left\langle\varpi_{k},\left[\varpi_{k}^{\vee}\right]\right\rangle$. Then the sequence

$$
0 \longrightarrow \pi_{1}(S) \longrightarrow \pi_{1}(L) \longrightarrow \pi_{1}(\bar{H}) \longrightarrow 0
$$

is isomorphic to the sequence

$$
0 \longrightarrow \mathbb{Z} \stackrel{d \cdot}{\longrightarrow} \mathbb{Z} \longrightarrow \mathbb{Z} / d \mathbb{Z} \longrightarrow 0 .
$$

The situation for non-simply connected groups turns out to be quite involved, from the algebra as well as from the topology. Since our main interest lies in the case where $G$ is simple and simply connected, we shall separate the two cases and treat the simply connected case first.

\section{§5. Simply Connected Simple Groups}

Let $G$ be simple and simply connected, and let $L=L_{k}$ be a maximal Levi subgroup $G$. According to Lemma 4.1, the group $L$ may be described as the quotient $\mathbb{C}^{*} \times^{\mu_{d}, \varphi} H$ of $\mathbb{C}^{*} \times H$ by a central cyclic subgroup $C_{d}=$ $\left\{\left(t, \varphi(t)^{-1}\right) \in \mathbb{C}^{*} \times H \mid t \in \mu_{d}\right\}$ associated with an embedding ('identification') $\varphi: \mu_{d} \longrightarrow C(H)$ of $\mu_{d}$ into the center of $H$. Note that, in the product $C(H)=\prod_{j=1}^{r} C\left(H_{j}\right)$, all factors $C\left(H_{j}\right)$ are cyclic subgroups $\mu_{d_{j}}$, except in case $D_{\text {even }}$, where we have $\mu_{2} \times \mu_{2}$. Thus, any component $\varphi_{j}$ of $\varphi$ can be written in the form $t \mapsto t^{m_{j}}$ (resp. $t \mapsto\left(t^{m_{j}}, t^{n_{j}}\right)$, if $H_{j}$ is of type $D_{\text {even }}$ ). Here, the $m_{j},\left(n_{j}\right), j=1, \ldots, r$, are integers satisfying $d_{j} m_{j} \equiv d_{j} n_{j} \equiv 0$ modulo $d$. 
They are uniquely determined modulo $d$ and up to outer automorphism of the factors $H_{j}$. Conversely, they determine $\varphi$ and thus $L$, up to isomorphism.

In the following discussion, we will refer to these data by the expression

$$
\left[m_{1}, \ldots, m_{r}\right]_{d}, \quad \text { resp. }\left[m_{1}, \ldots,\left(m_{j}, n_{j}\right), \ldots, m_{r}\right]_{d},
$$

which we shall call the symbol of the Levi subgroup L. Assuming the Dynkin diagrams in the form and with the numbering as in [5] or the tables below, the types of the simple factors $H_{j}$ of $L$ and their order in the symbol can be simply read off from their position in the Dynkin diagram $\Delta$, i.e. the left factor will correspond to the left component of $\Delta \backslash\left\{\alpha_{k}\right\}$, etc.

Finally note that, for any positive integer $\kappa$, the symbol $\left[\kappa m_{1}, \ldots, \kappa m_{r}\right]_{\kappa d}$ gives rise, in a similar way, to an abstract algebraic group $\mathbb{C}^{*} \times^{\mu_{\kappa d}, \varphi \circ \kappa} H$ isomorphic to $L$. Whereas the reconstruction of the groups $L$ as subgroups inside $G$ leads only to 'reduced' symbols (since $\mu_{d}$ embeds into $H$ ), we shall nonetheless make use of this fact for the purpose of notational simplification and uniformization.

In the table of the following theorem, we shall list the dimensions of the $P$-maximal $G$-bundles $\xi$ for all simple and simply connected $G$ and for all maximal $P \subset G$. This is done slightly differently for classical and exceptional types. In the classical case, one finds, above or in front of every node $\alpha_{k} \in \Delta$, the dimension $\operatorname{dim} \operatorname{Aut}_{L_{k}}\left(\xi_{L_{k}}\right)$ of the automorphism group of the canonical semistable reduction $\xi_{L_{k}}$ of $\xi$. Below or behind the node, one finds added (with an extra +-sign) the dimension $\operatorname{dim}_{\operatorname{Aut}_{G}}(\xi)^{+}$as given in Theorem 2.6. In the exceptional cases, these two numbers are always put together, below or behind the relevant node. Above or in front of the node, we find the symbol of the corresponding Levi subgroup $L_{k}$ from which we will compute $\operatorname{dim} \operatorname{Aut}_{L_{k}}\left(\xi_{L_{k}}\right)$ by means of Theorem 2.2 .

(5.1) Theorem. Let $G$ be simple and simply connected, $\alpha_{k}$ a simple root and $P_{k}$ the associated parabolic subgroup of $G$. Let $\xi$ be a $P_{k}$-maximal $G$ bundle. Then the dimension of its automorphism group $\operatorname{Aut}_{G}(\xi)$ is given in the following table.

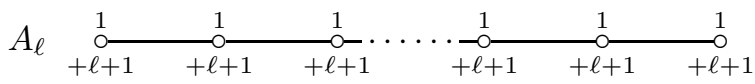

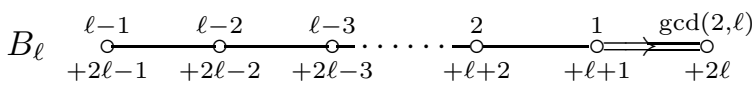




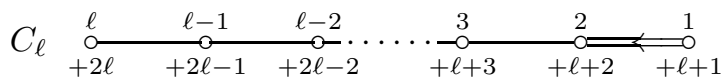

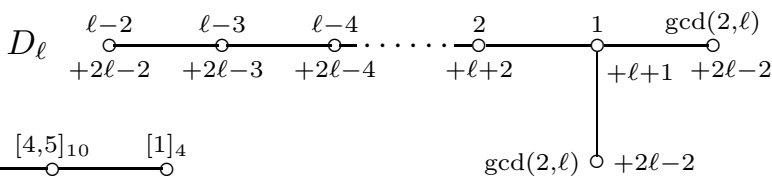

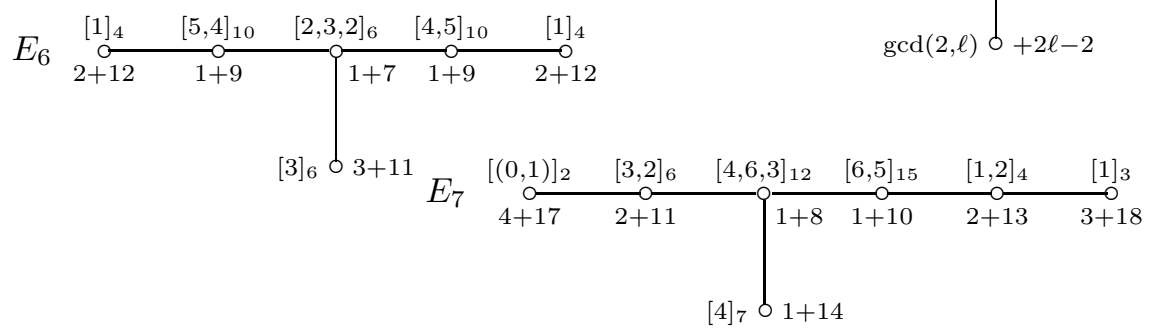

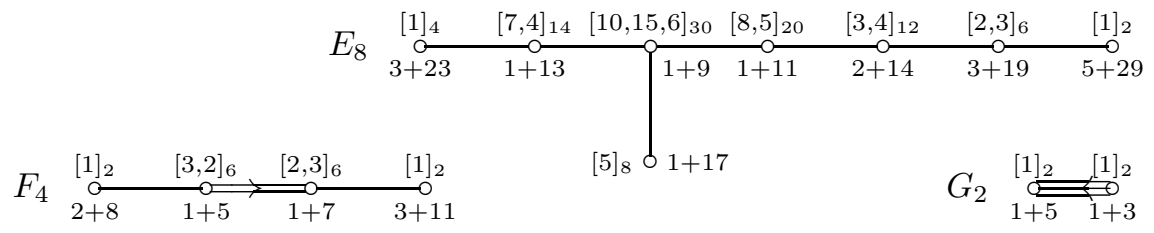

(5.2) Proof. Let $\xi$ be a $P_{k}$-maximal $G$-bundle with canonical semistable reduction $\left(L, \xi_{L}\right), L=L_{k}$. The formula $\operatorname{dim} \operatorname{Aut}_{G}(\xi)^{+}=\left\langle 2 \rho, \varpi_{k}^{\vee}\right\rangle /\left\langle\varpi_{k}, \varpi_{k}^{\vee}\right\rangle$ provided by Theorem 2.6 can be easily evaluated with the help of the tables in [5].

(5.2.1) The computation of $\operatorname{dim} \operatorname{Aut}_{L}\left(\xi_{L}\right)$ will be reduced to Theorem 2.2 as follows. Let us adhere to the previous notation as in 4.1, 4.3, 4.8, and the subsequent discussion. Then we have an exact sequence

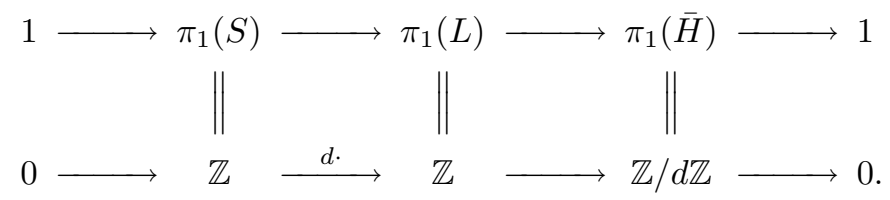

As fundamental group, we may identify $\mathbb{Z} / d \mathbb{Z}$ with the cyclic subgroup $S \cap$ $H=\varphi\left(\mu_{d}\right)$ in the center of $H$. Because of the realisation $L=\mathbb{C}^{*} \times{ }^{\mu_{d}, \varphi} H$, the generator $\overline{1}$ in $\mathbb{Z} / d \mathbb{Z}$ is then identified with the inverse of the generator $c=\varphi\left(e^{2 \pi i / d}\right)$.

By extension of structure group, $L \longrightarrow \bar{H}$, any semistable $L$-bundle $\eta$ of topological type $\gamma(\eta)=m \in \mathbb{Z}$ induces a semistable $\bar{H}$-bundle $\bar{\eta}=\eta \times{ }^{L} \bar{H}$ of topological type $\gamma(\bar{\eta})=\bar{m} \in \mathbb{Z} / d \mathbb{Z}$. Conversely, since $H^{2}\left(E, \underline{\mathbb{C}}_{E}^{*}\right)=0$, every 
semistable $\bar{H}$-bundle $\bar{\eta}$ reduces to a semistable $L$-bundle $\eta$, unique up to an action of $H^{1}\left(E, \mathbb{C}_{E}^{*}\right) \cong E \times \mathbb{Z}$ (cf. e.g. [11]). A comparison of the adjoint bundles $\operatorname{ad}(\eta)$ and $\operatorname{ad}(\bar{\eta})$ immediately shows

$$
\operatorname{dim} \operatorname{Aut}_{L}(\eta)=\operatorname{dim}_{\operatorname{Aut}_{\bar{H}}}(\bar{\eta})+1 .
$$

Assume that $\eta$, like $\xi_{L}$, is of minimal Chern class, i.e. $\gamma(\eta)=1$. Then the topological class of $\bar{\eta}$ is $\overline{1} \in \mathbb{Z} / d \mathbb{Z}$. Thus, the isomorphism class of $\bar{\eta}$ belongs to

$$
\mathcal{M}\left(\bar{H}, c^{-1}\right)=\mathcal{M}\left(H /\langle c\rangle, c^{-1}\right)=\prod_{j=1}^{r} \mathcal{M}\left(H_{j} /\left\langle c_{j}\right\rangle, c_{j}^{-1}\right),
$$

where we have expressed $c$ as a product $c=\prod_{j=1}^{r} c_{j}$ in $H=\prod_{j=1}^{r} H_{j}$. The dimension of $\operatorname{Aut}_{\bar{L}}(\bar{\eta})$ is now described in Theorem 2.2. In particular, for regular $\eta$, like $\xi_{L}$, we get the minimal value

$$
\operatorname{dim} \operatorname{Aut}_{L}(\eta)=\sum_{j=1}^{r} \ell_{c_{j}}+1,
$$

where $\ell_{c_{j}}+1$ is the number of $\left\langle c_{j}\right\rangle$-orbits on the extended Dynkin diagram of $H_{j}$, cf. Theorem 1.5.4 and its preceding explanations.

In the classical cases, the structure of the maximal Levi subgroups $L$ can be determined by direct inspection.

(5.2.2) CASE $A_{\ell}$. Let $n=\ell+1$. The $k$-th Levi subgroup $L_{k}$ may be written as $S\left(G L_{k} \times G L_{n-k}\right)=\left\{(g, h) \in G L_{k} \times G L_{n-k} \mid \operatorname{det}(g) \cdot \operatorname{det}(h)=1\right\}$. Its (possibly non-reduced) symbol with respect to the product $H_{k}=S L_{k} \times S L_{n-k}$ is $[n-k, k]_{k(n-k)}$, yielding the generating element $c=\left(e^{2 \pi i / k}, e^{2 \pi i / n-k}\right)$ of $S \cap H$ inside the center $C\left(H_{k}\right)=\mu_{k} \times \mu_{n-k}$. Let $\eta$ be a semistable $L_{k}$-bundle of minimal topological type $\gamma(\eta)=1$. Using Example 1.5.8, we get $0+0+1=1$ for the dimension of its automorphim group $\operatorname{Aut}_{L_{k}}(\eta)$.

(5.2.3) CASE $B_{\ell}$. Let $n=2 \ell+1$, and let us first look at the extremal case of $L_{\ell}$. The corresponding Levi subgroup $O L_{\ell}$ of the orthogonal group $S O_{n}$ has the simple form $O L_{\ell}=G L_{\ell}$. The preimage $L_{\ell} \subset S p i n_{n}$ is given as the double ('spin') cover ${ }_{s} G L_{\ell}=\left\{(g, s) \in G L_{\ell} \times \mathbb{C}^{*} \mid \operatorname{det}(g)=s^{2}\right\}$, which may be described by the symbol $[2]_{\ell}$. This corresponds to the central element $c=e^{4 \pi i / \ell}$ in $\mu_{\ell}=C\left(H_{\ell}\right)$. The dimension of $\operatorname{Aut}(\eta)$ for a regular semistable ${ }_{s} G L_{\ell}$-bundle $\eta$ with $\gamma(\eta)=1$ is thus $0+1$ or $1+1$, according to whether $\ell$ is odd or even. Let us now look at the remaining cases, $k<\ell$. There, the Levi subgroup $O L_{k} \subset S O_{n}$ admits a product decomposition $O L_{k}=G L_{k} \times S O_{n-2 k}$ of type 
$A_{k-1} \times B_{\ell-k}$. This is not preserved under pull-back into $S_{p i n}$. However, like in the case of spin groups for direct sums, the group $L_{k}$ is now a quotient $L_{k} \cong$ $\left({ }_{s} G L_{k} \times S p i n_{n-2 k}\right) / \mu_{2}$ of the individual spin covers ( $\mu_{2}$ diagonally embedded $\left.)\right)$. It can thus be described by the symbol $[2, k]_{2 k}$, corresponding to the central element $c=\left(e^{2 \pi i / k},-1\right) \in \mu_{k} \times \mu_{2}=C\left(H_{k}\right)$. This gives the dimension $0+(\ell-$ $k-1)+1=\ell-k$ for the group $\operatorname{Aut}_{L_{k}}(\eta)$ of a regular semistable $L_{k}$-bundle $\eta$ with $\gamma(\eta)=1$.

(5.2.4) CASE $C_{\ell}$. Here, the Levi subgroup $L_{k}$ is a product $G L_{k} \times S p_{2(\ell-k)}$ of type $A_{k-1} \times C_{\ell-k}$. Its symbol with respect to the product $H_{k}=S L_{k} \times S p_{2(\ell-k)}$ is given by $[1,0]_{k}$, corresponding to the central element $c=\left(e^{2 \pi i / k}, 1\right) \in \mu_{k} \times$ $\mu_{2}=C\left(H_{k}\right)$. The dimension of $\mathrm{Aut}_{L_{k}}(\eta)$ for a regular $L_{k}$-bundle is thus $0+(\ell-k)+1=\ell-k+1$.

(5.2.5) CASE $D_{\ell}$. This case is very similar to that of $B_{\ell}$. For the extremal indices $k=\ell-1, \ell$, the corresponding Levi subgroup $L_{k}$ is again ${ }_{s} G L_{\ell}$, leading to minimal dimensions 1 or 2 , according to the parity (odd/even) of $\ell$. In the remaining cases, $k<\ell-1$, the Levi subgroups $L_{k}$ are of semisimple type $A_{k-1} \times D_{\ell-k}$ and realized as quotients $L_{k} \cong\left({ }_{s} G L_{k} \times \operatorname{Spin}_{2(\ell-k)}\right) / \mu_{2}\left(\mu_{2}\right.$ diagonally embedded, also into the center $\mu_{2} \times \mu_{2} \subset \operatorname{Spin}_{2(\ell-k)}$, if $\ell-k$ is even). They are described by the symbol $[2, k]_{2 k}(\ell-k$ odd $)$ resp. $[2,(k, k)]_{2 k}$ $(\ell-k$ even $)$, corresponding to central elements $c=\left(e^{2 \pi i / k},-1\right) \in \mu_{k} \times \mu_{4}$ resp. $c=\left(e^{2 \pi i / k},-1,-1\right) \in \mu_{k} \times \mu_{2} \times \mu_{2}$ of $H_{k}$. This yields the minimal dimension $0+(\ell-k-2)+1=\ell-k-1$.

(5.2.6) To investigate the structure of $L_{k}$ in the exceptional cases, we employ a general method, which may also be used in the classical cases (it leads, however, to reduced symbols which, due to the underlying arithmetic, involve a greater number of case specifications). To determine the symbol of $L_{k}$, we look at irreducible representations $W_{j}$ of $L_{k}$ generated by the highest weight vectors $v_{j}$ in certain irreducible representations $V_{j}$ of $G$ with highest weight $\omega_{j}$. The action of the central torus $S_{k} \cong \mathbb{C}^{*}$ of $L_{k}$ on $W_{j}$ is then given by the weight $m=\left\langle\omega_{j},\left[\varpi_{k}^{\vee}\right]\right\rangle \in \mathbb{Z}$. Comparing this with the action of the center of $H_{k}$ on $W_{j}$, will allow an identification of the elements of $\mu_{d} \subset S_{k}$ with those in the center of $H_{k}$, provided we have looked at sufficiently many such representations $W_{j}$. This will be the case as soon as the direct sum $\bigoplus_{j} W_{j}$ is a faithful $H_{k}$-module. In most cases, choosing among the extremal fundamental dominant weights $\varpi_{1}, \varpi_{2}, \varpi_{\ell-1}, \varpi_{\ell}$ for $G$ will do (notation as in the tables of [5]). Using different weights or, alternatively, outer automorphisms, we have occasionally been able to obtain smaller exponents $m$ inside the symbol. 
Let us demonstrate this procedure in three examples.

(5.2.7) Let $G$ be of type $E_{8}$ with Levi subgroup $L_{4}$ of semisimple type $A_{2} \times A_{1} \times$ $A_{4}$. In this case, may identify roots and coroots, and we immediately get $\left[\varpi_{4}^{\vee}\right]=$ $\varpi_{4}^{\vee}=\varpi_{4}$ in $X_{*}(T)$. From the tables in [5], we read off $d=\left\langle\varpi_{4}, \varpi_{4}\right\rangle=30$. The fundamental $G$-representations with dominant weights $\varpi_{1}, \varpi_{2}$ and $\varpi_{8}$ will, on their respective top-level, induce the natural representations of the factors of $H_{4} \cong S L_{3} \times S L_{2} \times S L_{5}$. Thus their sum is faithful. The central subgroup $S_{4} \cong \mathbb{C}^{*} \subset L_{4}$ will act on these modules by means of the weights $\left\langle\varpi_{1}, \varpi_{4}\right\rangle=10$, $\left\langle\varpi_{2}, \varpi_{4}\right\rangle=15$, and $\left\langle\varpi_{8}, \varpi_{4}\right\rangle=6$, yielding the symbol $[10,15,6]_{30}$ and the central element $c=\left(e^{2 \pi i / 3}, e^{\pi i}, e^{2 \pi i / 5}\right)$ in the center $\mu_{3} \times \mu_{2} \times \mu_{5}$ of $H_{4}$. The minimal dimension of $\operatorname{Aut}(\eta)$ for a semistable $L_{4}$-bundle $\eta$ with $\gamma(\eta)=1$ is thus $0+0+0+1=1$.

(5.2.8) Next, consider $G$ of type $E_{7}$ with Levi subgroup $L_{1}$ of semisimple type $D_{6}$. Again, we may identify roots and coroots, and we see from the tables in [5] that $\left[\varpi_{1}^{\vee}\right]=\varpi_{1}^{\vee}=\varpi_{1} \in X_{*}(T)$. Furthermore, $\left\langle\varpi_{1}, \varpi_{1}\right\rangle=2$. The fundamental $G$-representations with dominant weights $\varpi_{2}$ and $\varpi_{3}$ will, on their top-levels, induce the two half-spin representations $\Delta_{+}$and $\Delta_{-}$on $H_{1} \cong \operatorname{Spin}_{12}$, whose sum, $\Delta_{+} \oplus \Delta_{-}$, realizes a faithful representation of $\operatorname{Spin}_{12}$. Restricted to the center $\mu_{2} \times \mu_{2}$ of $\operatorname{Spin}_{12}$, the two representations $\Delta_{+/-}$realize the two projections. The central torus $S_{1} \cong \mathbb{C}^{*}$ of $L_{1}$ acts on these modules by the weights $\left\langle\varpi_{2}, \varpi_{1}\right\rangle=2$ and $\left\langle\varpi_{3}, \varpi_{1}\right\rangle=3$. Restriction to the subgroup $\mu_{2} \subset$ $S_{1}$ now yields the symbol $[(0,1)]_{2}$ and the corresponding central element in $c=(1,-1) \in \mu_{2} \times \mu_{2}=C\left(H_{1}\right)$. The minimal dimension of $\operatorname{Aut}(\eta)$ for a semistable $L_{1}$-bundle $\eta$ with $\gamma(\eta)=1$ is thus $3+1=4$ (the extended Dynkin diagram of type $D_{6}$ is just folded once over its midpoint by the automorphism corresponding to the element $c$ ).

(5.2.9) Finally, let us look at $G$ of type $E_{6}$ with Levi subgroup $L_{5}$ of semisimple type $A_{4} \times A_{1}$. Again, we identify roots and coroots. This time, we find from the tables of [5] that $\left[\varpi_{5}^{\vee}\right]=3 \varpi_{5}^{\vee}=3 \varpi_{5}$ in the 'simply connected' coroot lattice $X_{*}(T)$ and that $d=\left\langle\varpi_{5},\left[\varpi_{5}^{\vee}\right]\right\rangle=10$. The fundamental $G$-representations with dominant weights $\varpi_{1}$ and $\varpi_{6}$ will, on their top-levels, induce the natural representations of the factors of $H_{5} \cong S L_{5} \times S L_{2}$. Thus their sum is faithful. The central subgroup $S_{5} \cong \mathbb{C}^{*} \subset L_{5}$ will act on these modules by means of the weights $\left\langle\varpi_{1},\left[\varpi_{5}^{\vee}\right]\right\rangle=4$ and $\left\langle\varpi_{6},\left[\varpi_{5}\right]^{\vee}\right\rangle=5$, yielding the symbol $[4,5]_{10}$ and the central element $c=\left(e^{4 \pi i / 5},-1\right)$ in the center $\mu_{5} \times \mu_{2}=C\left(H_{5}\right)$. The minimal dimension of $\operatorname{Aut}(\eta)$ for a semistable $L_{5}$-bundle $\eta$ with $\gamma(\eta)=1$ is now $0+0+1=1$. 
(5.3) Definition. An unstable $G$-bundle $\xi$ of topological type $\gamma \in \pi_{1}(G)$ is called regular if its automorphism group $\operatorname{Aut}_{G}(\xi)$ has minimal dimension among all unstable $G$-bundles of topological type $\gamma$.

(5.4) Remark. This definition is similar to the definition for semistable bundles 2.1. However, as seen below, there is a discrepancy in the dimensions of $\operatorname{Aut}_{G}(\xi)$ for regular semistable and regular unstable bundles. To 'correct' this fact, one has to regard G-bundles as parametrized by extended loop groups with conjugacy as equivalence, as we will do in subsequent work, cf. [13], [14] for an outline. Then the elements representing regular, resp. subregular, bundles $\xi$ will acquire the same stabilizer dimension, irrespective of their stability nature.

The following observations are already related to this loop group context. Note that the elliptic curve $E$ acts on the set of all bundles by pull back via translations in the base $E$. Let $\xi$ be an unstable $G$-bundle with canonical reduction $\left(L, \xi_{L}\right)$. Due to the unicity (up to isomorphism) of the canonical reduction, the association $\xi \mapsto \xi_{L}$ is compatible with translation. In particular, since $\xi_{L}$ has non-zero degree, $\xi$ can be isomorphic to only finitely many of its E-translates.

(5.5) Definition. Two $G$-bundles $\xi$ and $\zeta$ are called extended isomorphic if they become isomorphic as $G$-bundles after possible translation in the base $E$.

(5.6) Theorem. Let $G$ be simple and simply connected.

(a) An unstable G-bundle $\xi$ is regular exactly when $\operatorname{dim}_{\operatorname{Aut}_{G}}(\xi)=\ell+2$.

(b) For $G=S L_{n}$ there are exactly $n-1$ distinct extended isomorphism classes of regular unstable bundles $\xi$. They are in bijection with the $n-1$ distinct conjugacy classes of maximal Levi subgroups $L_{k}$ of $G$ by means of their canonical reduction $\left(L_{k}, \xi_{L_{k}}\right)$.

(c) If $G$ is not isomorphic to $S L_{n}$, then, up to extended isomorphism, there exists a unique regular unstable $G$-bundle.

(5.7) Proof. The description in 5.1 gives a list of the minimal dimensions of the automorphism groups of unstable bundles $\xi$ whose parabolic type $P_{k}$ is maximal. This leaves only one candidate for $k$, except in case $A_{\ell}$. In all cases, the degeneration results, Propositions 3.3 and 3.9, imply then that the Levi subgroup of the canonical reduction of a regular unstable bundle cannot have a semisimple rank less than $\ell-1$. The (relative) unicity of regular unstable 
$G$-bundles $\xi$ is seen as follows. The dimension $\operatorname{dim} \operatorname{Aut}_{L}\left(\xi_{L}\right)=1$ is also the dimension of the moduli space $\mathcal{M}\left(L, \gamma\left(\xi_{L}\right)\right)$ of all canonical reductions of unstable bundles of the same type $\mu(\xi)$ as $\xi$. Since, by translation, the compact group $E$ acts non-trivially on that compact and connected space, it must consist of a single orbit.

(5.8) Remark. Note that, here as well as in later analogous arguments, the exclusion of unsuitable parabolic subgroups $P_{k}$ requires only the knowledge of the easily accessible dimensions of $\operatorname{Aut}_{G}(\xi)^{+}$. Of course, for the correct candidates, we have to know $\operatorname{dim} \operatorname{Aut}_{L}\left(\xi_{L}\right)$ as well. In addition, the corresponding moduli space of $L$-bundles is of relevance in our geometric investigations, [13], $[14]$.

(5.9) Remark. An announcement of this result was given first in [10].

(5.10) Example. In [10], one also finds 'explicit' constructions for the regular bundles. In case of the classical groups, they are realized in terms of the vector bundles associated to the natural representations.

(5.10.1) Here is the case $G=S L_{n}$. Let $0<k<n$ be an integer and $U$ a stable vector bundle of rank $k$ and $\operatorname{degree} \operatorname{deg}(U)=1$. Then there is a unique stable vector bundle $U^{\prime}$ of rank $n-k$ and degree -1 whose determinant line bundle $\bigwedge^{n-k} U^{\prime}$ is the inverse of that of $U$ (cf. e.g. [1]). The principal $G$-bundle $\xi$ derived from $U \oplus U^{\prime}$ is then a regular unstable bundle with canonical Levi subgroup $L_{k}=S\left(G L_{k} \times G L_{n-k}\right)$.

(5.10.2) In the case of an exceptional group, we can reformulate the constructions of [10] in a somewhat more systematic way which, suitably modified, works in all corank-1-cases. We shall restrict ourselves to the four cases where the Dynkin diagram has three branches, $D_{\ell}, E_{6}, E_{7}, E_{8}$. Then the derived subgroup $H$ of the canonical Levi subgroup $L$ is a product $H=S L_{p} \times S L_{q} \times S L_{r}$, $p, q, r$ denoting the lengths of the branches. By exploiting the (explicit) symbol of $L$, one easily sees that the group $L$ may be realized as the subgroup of a product of three general linear groups

$$
L=\left\{(A, B, C) \in G L_{p} \times G L_{q} \times G L_{r} \mid \operatorname{det}(A)=\operatorname{det}(B)=\operatorname{det}(C)\right\} .
$$

From the long exact sequence of cohomology sets attached to the exact sequence of group sheaves over $E$

$$
1 \longrightarrow \underline{L} \longrightarrow \underline{G L}_{p} \times \underline{G L}_{q} \times \underline{G L}_{r} \longrightarrow \underline{\mathbb{C}}^{*} \times \underline{\mathbb{C}}^{*} \longrightarrow 1
$$


we see that any $L$-bundle $\eta$ is given by a unique $G L_{p} \times G L_{q} \times G L_{r}$-bundle $\left(\eta_{p}, \eta_{q}, \eta_{r}\right)$ such that $\operatorname{det}\left(\eta_{p}\right)=\operatorname{det}\left(\eta_{q}\right)=\operatorname{det}\left(\eta_{r}\right)$. In particular, a bundle of minimal degree has to satisfy $\operatorname{deg}\left(\eta_{p}\right)=\operatorname{deg}\left(\eta_{q}\right)=\operatorname{deg}\left(\eta_{r}\right)=1$. If we choose the direct sum of the three natural representations $V=\mathbb{C}^{p} \oplus \mathbb{C}^{q} \oplus \mathbb{C}^{r}$ as a faithful module for $L$, then the associated vector bundle $\eta \times{ }^{L} V$ is a direct sum $U_{p} \oplus U_{q} \oplus U_{r}$ of stable vector bundles $U_{s}$ with $\operatorname{rank}\left(U_{s}\right)=s$ and with all determinant line bundles equal, of degree 1 . This realisation illuminates the unicity statement. To get a $G$-bundle, however, one still has to extend the bundle $\eta$ to $G$. The adjoint bundle ad $(\xi)$ can then be obtained from $\eta \times{ }^{L} V$ by applying suitable tensor functors to $V$. For example, the representation of $L$ on $\mathfrak{g}(1)$ (the lowest positively graded piece of $\mathfrak{g}$ with respect to $\mu(\xi)$ ) is given by $V(1)=\mathbb{C}^{p} \otimes \mathbb{C}^{q} \otimes\left(\mathbb{C}^{r}\right)^{*}$, where $r=2$.

Let us now turn to the more involved case of subregular bundles.

(5.11) Definition. An unstable $G$-bundle $\xi$ of topological type $\gamma \in \pi_{1}(G)$ is called subregular if its automorphism group $\operatorname{Aut}_{G}(\xi)$ has dimension $\operatorname{dim} \operatorname{Aut}_{G}\left(\xi_{r}\right)+2$ where $\xi_{r}$ is a regular unstable $G$-bundles of topological type $\gamma$.

(5.12) Theorem. Let $G$ be simple and simply connected. There are no unstable $G$-bundles $\xi$ with $\operatorname{dim} \operatorname{Aut}_{G}(\xi)=\ell+3$, and we have $\operatorname{dim} \operatorname{Aut}_{G}(\xi)=\ell+4$ for all subregular unstable $G$-bundles $\xi$. Up to extended isomorphism, there are the following subregular unstable $G$-bundles.

(a) If $G$ is of type $A_{1}, E_{7}, E_{8}, F_{4}$ or $G_{2}$, there is a unique subregular bundle.

(b) If $G$ is of type $E_{6}$, there are exactly two subregular unstable bundles permuted by the outer automorphism.

(c) If $G=S L_{n}, n>2$ : There are $n-2$ disjoint families of subregular unstable bundles, each parametrized by the elliptic curve E. They are in bijection with the $n-2$ subsets $\{k, k+1\} \subset\{1, \ldots, n-1\}$ by means of their canonical reduction $\left(L, \xi_{L}\right)$, in which $L$ is a Levi subgroup of corank 2 conjugate to $L_{\Delta \backslash\left\{\alpha_{k}, \alpha_{k+1}\right\}}$.

(d) If $G$ is of type $B_{\ell}$ with $\ell \neq 3$ or of type $C_{\ell}$ or of type $D_{\ell}$ with $\ell>5$, there is a family of subregular unstable bundles parametrized by the projective line $\mathbb{P}^{1}$.

(e) If $G$ is of type $D_{4}$, there are three disjoint families of subregular unstable bundles, each parametrized by the projective line $\mathbb{P}^{1}$.

(f) If $G$ is of type $B_{3}$, resp. $D_{5}$, there is a family of subregular unstable bundles parametrized by the projective line $\mathbb{P}^{1}$. In addition, there are one, 
resp. two more subregular bundles which are not adjacent to the first mentioned family.

(5.13) Proof. The strategy of proof is the same as in the regular case. Except for the case of $S L_{n}$, one finds from 5.1 the correct Levi subgroups, unicity or type of families for unstable bundles of minimal degree 1. Using Propositions 3.3 and 3.9, one excludes non-maximal Levi subgroups. The statement about the non-adjacency in the $D_{5}$-case already appeared in the example to Theorem 3.5. The case $B_{3}$ is settled similarly.

It remains to treat the case of $S L_{n}$. Here, the group $S L_{2}$ is a special case by itself. In fact, since for an unstable $G$-bundle $\operatorname{dim}^{\operatorname{Aut}_{G}}(\xi)^{+}$is larger than a positive multiple of $\ell+1$, this is the only case, where an $L$-bundle of degree 2 can lead to a subregular bundle. The associated rank- 2 vector bundle is then $U \oplus U^{*}$, where $U$ is a line bundle of degree 2. For $n>2$ we have to use corank-2 Levi subgroups $L$ of $S L_{n}$. The vector bundles $\mathrm{U}$ associated naturally to corresponding maximal unstable $S L_{n}$-bundles $\xi$ decompose into a direct sum of 3 summands, say

$$
U=U_{1} \oplus U_{2} \oplus U_{3}, \operatorname{rank}\left(U_{i}\right)=n_{i}, \operatorname{deg}\left(U_{i}\right)=d_{i}, \sum_{i} n_{i}=n, \sum_{i} d_{i}=0 .
$$

Assuming a decreasing sequence of slopes $d_{i} / n_{i}$ and $d_{2} \geqslant 0$ (otherwise, use the dual bundle), one computes the values $\operatorname{dim} \operatorname{Aut}_{S L_{n}}(\xi)^{+}=\left(n+n_{2}\right) d_{1}+\left(n-n_{1}\right) d_{2}$ and $\left.\operatorname{dim} \operatorname{Aut}_{L}\left(\xi_{L}\right)\right) \geqslant 2$. This leaves the only choice: $d_{1}=1, d_{2}=0, d_{3}=-1$ and $n_{2}=1$. From that, our respective assertions follow.

(5.14) Remark. Whenever one of the families discussed above is parametrized by $\mathbb{P}^{1}$, this is a consequence of the relevant moduli space $\mathcal{M}\left(\bar{H}, c^{-1}\right)$ of semistable $\bar{H}$-bundles being a weighted projective space of dimension 1 . At first sight, the parametrization is only set-theoretical. However, as shown in [10] in the case of simply connected $\bar{H}$, this family may be realized holomorphically by exploiting a $\mathbb{C}^{*}$-equivariant 'transversal slice' for a regular unstable $\bar{H}$-bundle. For general semisimple $\bar{H}$, the same procedure can be applied. The existence problem for regular unstable bundles is settled in our next section.

\section{§6. Non-Simply Connected Simple Groups}

In this section, we want to deal with the general but more involved case of non-simply connected groups $G$. Let $\xi$ be a $P$-maximal $G$-bundle with canonical reduction $\left(L, \xi_{L}\right)$, where $P=P_{k}$ is a maximal parabolic with Levi subgroup 
$L=L_{k}$. The strategy for computing the dimension of $\operatorname{Aut}_{G}(\xi)$ is the same as in the simply connected case. Theorem 2.6 gives a formula for $\operatorname{dim}_{\operatorname{Aut}_{G}}(\xi)^{+}$, and $\operatorname{dim}_{\operatorname{Aut}_{L}}\left(\xi_{L}\right)$ is reduced to Theorem 2.2 by means of the exact sequence

$$
1 \longrightarrow \pi_{1}(S) \longrightarrow \pi_{1}(L) \longrightarrow \pi_{1}(\bar{H}) \longrightarrow 1 .
$$

All semistable $L$-bundles $\eta$ extend to semistable $\bar{H}$-bundles $\bar{\eta}$ and, conversely, any semistable $\bar{H}$-bundle $\bar{\eta}$ reduces to a semistable $L$-bundle $\eta$ unique up to an action of $E \times \mathbb{Z}$. The topological class $\gamma(\eta) \in \pi_{1}(L)$ is mapped to $\gamma(\bar{\eta}) \in \pi_{1}(\bar{H})$ under the above surjection, and $\operatorname{dim}_{\operatorname{Aut}_{L}}(\eta)=\operatorname{dim}_{\operatorname{Aut}_{\bar{H}}}(\bar{\eta})+1$. The problem lies mainly in the determination of the relation between the topological type of $\xi$ and that of $\bar{\eta}$, which is now complicated by the interference of the fundamental groups of $G$ and $H$.

$$
\begin{gathered}
\pi_{1}(\bar{H}) \longleftarrow \pi_{1}(L) \longrightarrow \pi_{1}(G) \\
\gamma(\bar{\eta}) \longleftarrow \gamma(\eta) \longrightarrow \gamma(\xi)
\end{gathered}
$$

(6.1) In principle, the structure of $L$ could still be described by a symbol like in the simply connected case. However, the center $C(H)$ of $H$ may not split any more according to the simple factors of $H$. On the other hand, for adjoint $G$, the group $C(H)$ itself is cyclic and isomorphic to $S \cap H$. Because of the more involved relations between the various fundamental groups, we will essentially proceed by a case-by-case analysis. Some general observations may nonetheless precede the individual computations.

(6.1.1) We may restrict our considerations to groups of adjoint type. Let $G$ be a semisimple group with projection $\pi: G \longrightarrow G^{a d}$ onto its adjoint group $G^{a d}$. Via $\pi$, any $G$-bundle $\xi$ extends automatically to a $G^{a d}$-bundle $\bar{\xi}$, preserving the

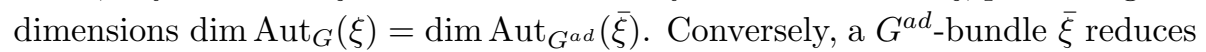
to a $G$-bundle $\xi$ exactly when its topological class $\gamma(\bar{\xi})$ lies in the subgroup $\pi_{*}\left(\pi_{1}(G)\right) \subset \pi_{1}\left(G^{a d}\right)$.

(6.1.2) Assume that $G$ is simple of adjoint type with Lie algebra $\mathfrak{g}$. The action of $S=S_{k} \cong \mathbb{C}^{*}$ provides $\mathfrak{g}$ with a $\mathbb{Z}$-grading, $\mathfrak{g}=\bigoplus_{m \in \mathbb{Z}} \mathfrak{g}(m)$. Then $L=$ $L_{k}$ acts on the graded components $\mathfrak{g}(m)$ by the restricted adjoint action, and $\mathfrak{g}(0)=\operatorname{Lie}(L)$. It is easily seen, (cf. e.g. [16, Proposition 8.6]), that $\mathfrak{g}(-1)$ is an irreducible $L$-module with heighest weight $-\alpha_{k}$. Moreover, it is a faithful $L$-module since, together with $\mathfrak{g}(0)$ and its contragredient $\mathfrak{g}(1)$, it generates $\mathfrak{g}$. This provides an identication of $S \cap H \cong \mu_{d} \subset \mathbb{C}^{*}$ with the full center $C(H)$ of $H$. In particular, the quotient group $\bar{H}=H / S \cap H$ is of adjoint type.

From the topological point of view, there are three different situations 
which we will encounter; recall that the fourth sequence in 4.3 gives an embedding of $\pi_{1}(H)$ into $\pi_{1}(G)$ :

$$
\text { (I) } \pi_{1}(H)=0, \quad(\mathrm{II}) \quad \pi_{1}(H)=\pi_{1}(G), \quad(\mathrm{III}) \quad 0 \neq \pi_{1}(H) \neq \pi_{1}(G) .
$$

The third situation (III) can only arise if the order of $\pi_{1}(G)$ is not a prime. For simple $G$, this can happen only if $G$ is of type $A$ or $D$, and we refrain from developing any generalities. In the diagrams, the nodes of type (I), (II), (III), will be marked by a $\circ$, a $\circledast$, or a $\odot$, respectively.

(6.1.3) $\pi_{1}(H)=0$ (I). This is closest to the simply connected case. According to Lemma 4.1, the intersection $S \cap H$ is a cyclic group of order $d=\left\langle\left[\varpi_{k}\right],\left[\varpi_{k}^{\vee}\right]\right\rangle$, and because of $\pi_{1}(H)=0$, the degree map $\pi_{1}(L) \longrightarrow \pi_{1}(\bar{S})$ induces now an isomorphism $\mathbb{Z} \longrightarrow \mathbb{Z}$, and the second exact sequence from 4.3 reads

$$
0 \longrightarrow \mathbb{Z} \stackrel{d}{\longrightarrow} \mathbb{Z} \longrightarrow \mathbb{Z} / d \mathbb{Z}=\pi_{1}(\bar{H}) \longrightarrow 0 .
$$

Thus, if $\eta$ is an $L$-bundle of degree $m$, the topological class $\gamma(\bar{\eta})$ in $\pi_{1}(\bar{H})=$ $\mathbb{Z} / d \mathbb{Z}$ is $\bar{m}$. Let $\xi$ be the $G$-extension of $\eta$. The third sequence of 4.3 tells us already that the topological class of $\xi$ is the residue class of $m$ in $\pi_{1}(G) \cong \mathbb{Z} / e \mathbb{Z}$, where $e=\left\langle\left[\varpi_{k}\right], \alpha_{k}^{\vee}\right\rangle$. In many cases, i.e. if $\pi_{1}(G)$ is isomorphic to $\mathbb{Z} / 2 \mathbb{Z}$, this will suffice for the determination of $\gamma(\xi)$. In the other cases, however, we have to relate this to a fixed isomorphism, i.e. $\pi_{1}(G)=X_{*}(T) / Q^{\vee}$. For that, we have to compute the residue class of $\left[\varpi_{k}^{\vee}\right] / d \in X_{*}(T) / Q^{\prime} \vee$ in $X_{*}(T) / Q^{\vee}$. The topological class of $\xi$ is then the $m$-fold of this element.

Because of $\pi_{1}(L)=0$, one may also describe $L$ and the resulting topological invariants somewhat more precisely by means of a symbol as in 5 and 5.1 (however, since the element $c=\varphi\left(e^{2 \pi i / d}\right)$ will always be a generator of the cyclic group $C(H)$, we effectively don't need that precision).

(6.1.4) $\pi_{1}(H)=\pi_{1}(G)(\mathrm{II})$. This condition implies that the kernel $C \cong \pi_{1}(G)$ of the universal covering $\tilde{G} \longrightarrow G$ is contained in the analogous subgroup $\tilde{H} \subset \tilde{G}$ which, in turn, implies $\left[\varpi_{k}\right]=\varpi_{k}$. The degree map, now given by $\varpi_{k}$, induces an isomorphism of $\mathbb{Z} \alpha_{k}^{\vee} \subset \pi_{1}(L)$ with $\mathbb{Z}=\pi_{1}(\bar{S})$ and thus a natural splitting

$$
\pi_{1}(L)=\mathbb{Z} \alpha_{k}^{\vee} \times \pi_{1}(G),
$$

such that the natural map $\pi_{1}(L) \longrightarrow \pi_{1}(G)$ identifies with the second projection. In particular, the topological type of the $G$-extension $\xi$ of an $L$-bundle $\eta$ does not depend on the degree of $\eta$. The degree of $\eta$ enters, however, into the topological type of the $\bar{H}$-extension $\eta$ according to the the surjection

$$
\pi_{1}(L)=\mathbb{Z} \alpha_{k}^{\vee} \times \pi_{1}(H) \longrightarrow \pi_{1}(\bar{H}) .
$$


The image of $\pi_{1}(H)$ in $\pi_{1}(\bar{H})$ is usually easy to describe. To determine the image of $\alpha_{k}^{\vee}$, we have to evaluate its class in $\pi_{1}(\bar{H})=X_{*}(T) /\left(Q^{\prime} \vee+\mathbb{Z}\left[\varpi_{k}^{\vee}\right]\right)$. For example, if $X_{*}(T)=P^{\vee}$, the class of $\alpha_{k}^{\vee}$ is a negative sum of 'neighbouring' fundamental co-weights $\varpi_{j}^{\vee},\left\langle\alpha_{j}, \alpha_{k}^{\vee}\right\rangle \neq 0$.

Let us now investigate the individual cases. Since the behaviour of regular and subregular unstable bundles varies strongly between different cases, we have not collected that in a separate statement. Instead, in each case, we will directly record the relevant properties. We remind the reader that, for ruling out unsuitable candidates for regular and subregular bundles, essentially the knowledge of the dimensions of $\operatorname{Aut}_{G}(\xi)^{+}$is sufficient.

(6.2) The CASE $A_{\ell}, G=P G L_{n}, n=\ell+1$. This is the adjoint group of type $A_{\ell}$ with fundamental group $\mathbb{Z} / \ell \mathbb{Z}$. We shall identify co-weights and weights and $\mathbb{Z} / \ell \mathbb{Z}$ with $P / Q$, in such a way, that the element $\bar{k}$ corresponds to the class of the $(n-k)$-th fundamental weight $\varpi_{n-k}$. The reduced rank $\ell_{\bar{k}}$ is $g-1$, where $g=(k, n)=(n-k, n)$. For the fundamental dominant weights, we have

$$
\left[\varpi_{k}\right]=(n / g) \varpi_{k} \quad \text { and } \quad d_{k}=\left\langle\left[\varpi_{k}\right], \varpi_{k}^{\vee}\right\rangle=k(n-k) / g,
$$

cf. also the Example following Theorem 2.6. The Levi subgroup $L_{k}$ is the quotient of $S\left(G L_{k} \times G L_{n-k}\right)$ by the full center $\mu_{n}$, and its semisimple subgroup $H_{k}$ is the quotient of $S L_{k} \times S L_{n-k}$ by $\mu_{g} \subset \mu_{n}$. Thus, we have $\pi_{1}\left(H_{k}\right) \cong \mathbb{Z} / g \mathbb{Z}$, and we are in Situation I exactly when $g=(k, n)=1$. Otherwise, we meet Situation III. The following explicit forms of the fundamental groups and their relating maps will be useful.

The fundamental group $\pi_{1}\left(L_{k}\right)=P / Q^{\prime} \cong \mathbb{Z} \times \mathbb{Z} / g \mathbb{Z}$ of $L_{k}$ may be written in lattice form

$$
P / Q^{\prime}=\left(\mathbb{Z} \varpi_{1} \oplus \mathbb{Z} \varpi_{\ell}\right) /\left\langle k \varpi_{1}-(n-k) \varpi_{\ell}\right\rangle=\left(\mathbb{Z} \alpha_{k} \oplus \mathbb{Z} \varpi_{\ell}\right) /\left\langle k \alpha_{k}-n \varpi_{\ell}\right\rangle
$$

(the equivalence of these two realisations follows from the identity (of classes in $\left.\left.P / Q^{\prime}\right) \alpha_{k}=\varpi_{1}+\varpi_{\ell}\right)$. Note that under the degree map to $\mathbb{Z}=\pi_{1}(\bar{S})$, the elements $\alpha_{k}$ and $\varpi_{\ell}$ are mapped to $n / g$ and $k / g$. Under the surjection $\pi_{1}\left(L_{k}\right) \longrightarrow \pi_{1}(G)$, the element $\varpi_{\ell}$ is mapped to $\overline{1} \in \mathbb{Z} / n \mathbb{Z}$, and, of course, $\alpha_{k}$ goes to $\overline{0}$. We shall make the identification $\pi_{1}\left(\bar{H}_{k}\right)=P / Q^{\prime}+\mathbb{Z} \varpi_{k}=$ $\mathbb{Z} / k \mathbb{Z} \times \mathbb{Z} /(n-k) \mathbb{Z}$ such that the class of $\varpi_{1}$ corresponds to $(\overline{1}, \overline{0})$ and that of $\varpi_{\ell}$ to $(\overline{0}, \overline{1})$. Then, the class of the element $\alpha_{k}$ corresponds to $(\overline{1}, \overline{1})$.

Assume that $\xi$ is an unstable $G$-bundle of topological type $\gamma(\xi)=\bar{m}$ in the fundamental group $\pi_{1}(G)=\mathbb{Z} / n \mathbb{Z}$ with canonical $L_{k}$-reduction $\eta$. Then the topological type of $\eta$ has to be of the form $\gamma(\eta)=u \alpha_{k}+m \varpi_{\ell}$, where $u$ is 
an arbitrary integer and $m \in \mathbb{Z}$ is a lift of $\bar{m}$. This gives $\operatorname{deg}(\eta)=(m k+u n) / g$ and, as a consequence of Theorem 2.6, $\operatorname{dim}^{\operatorname{Aut}_{G}}(\xi)^{+}=m k+u n$. For fixed $k$ and $m$, the minimal such dimension is given as the positive residue of $k m$ modulo $\mathrm{n}$ or $n$, if that residue is 0 . For the computation of $\operatorname{dim}_{A^{\prime}} \operatorname{cut}_{L_{k}}(\eta)$, we have to identify the image of $\gamma(\eta)$ in $\pi_{1}\left(\bar{H}_{k}\right)$. Writing $\gamma(\eta)$ in the above form, we obtain $(\bar{u}, \bar{u}+\bar{m})$ in $\mathbb{Z} / k \mathbb{Z} \times \mathbb{Z} /(n-k) \mathbb{Z}$.

Here is a list of the dimensions of the automorphism groups of maximal unstable bundles in some (essentially low rank) cases. Note that tables for $\gamma(\xi)=-\bar{k}$ are obtained from those for $\gamma(\xi)=\bar{k}$ by applying the diagram symmetry.

If the topological class of the unstable $G$-bundle $\xi$ is $\bar{m}=\overline{1}$, we can make the following general observation. For a reducing $L_{k}$-bundle $\eta$ of minimal degree, we get $\gamma(\eta)=0 \alpha_{k}+1 \varpi_{\ell}$ and, accordingly, $\operatorname{dim} \operatorname{Aut}_{G}(\xi)^{+}=\operatorname{deg}(\eta)=k$. The type of $\gamma(\bar{\eta})$ in $\mathbb{Z} / k \mathbb{Z} \times \mathbb{Z} /(n-k) \mathbb{Z}$ will be $(\overline{0}, \overline{1})$. Thus, we also get $\operatorname{dim} \operatorname{Aut}_{L_{k}}(\eta)=k$.

$A_{\ell}, P G L_{n}, n=\ell+1$

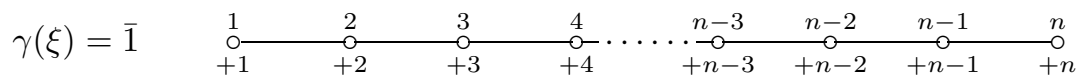

$A_{3}, P G L_{4}$

$$
\gamma(\xi)=\overline{2}
$$

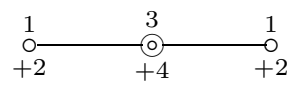

$A_{4}, P G L_{5}$

$$
\gamma(\xi)=\overline{2}
$$

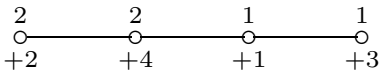

$A_{5}, P G L_{6}$

$$
\begin{aligned}
& \gamma(\xi)=\overline{2} \\
& \gamma(\xi)=\overline{3}
\end{aligned}
$$

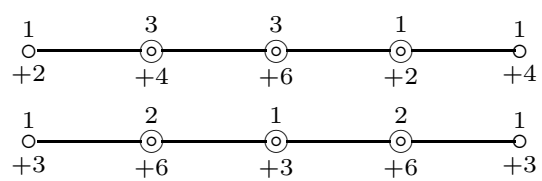

$A_{6}, P G L_{7}$

$$
\begin{aligned}
& \gamma(\xi)=\overline{2} \\
& \gamma(\xi)=\overline{3}
\end{aligned}
$$

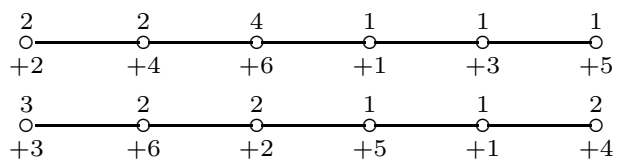

$A_{7}, P G L_{8}$

$$
\begin{aligned}
& \gamma(\xi)=\overline{2} \\
& \gamma(\xi)=\overline{3}
\end{aligned}
$$

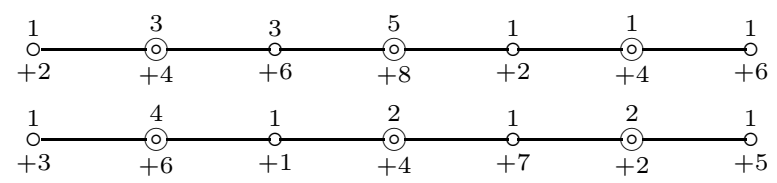




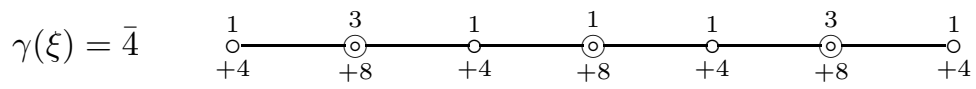

The behaviour of regular and subregular unstable $G$-bundles $\xi$ is most involved for this group, $G=P G L_{n}$. In each individual case, the sought for information can be extracted from our explicit formulae. We shall only give some relevant indications. Let $\gamma(\xi)=\bar{m}$ for some $0<m<n$ and $g=(m, n)$.

The regular case is still easy to overlook. Note that there are integers $u, k \in \mathbb{Z}$ such that $u n+k m=g$, and where $k$ is well determined up to multiples of $n / g$. Thus there are $g$ choices for $k$ satisfying $0<k<n$ and, correspondingly, $g$ distinct Levi subgroups $L_{k}$ with semistable bundles $\eta_{k}$ satisfying $\operatorname{dim} \operatorname{Aut}_{G}\left(\eta_{k} \times{ }^{L_{k}} G\right)=g$ (the minimal positive value attainable for $G$-bundles $\xi$ of type $\bar{m})$. We claim that also $\operatorname{dim} \operatorname{Aut}_{L_{k}}\left(\eta_{k}\right)$ attains the minimal value, 1. This follows from $(u, k)=1$ and $(u+m, n-k)=1$. The first equality is a consequence of $u n+k m=g$ and $(m, n)=g$, and the second is an easy consequence of the first (rewrite $u n+k m=g$ as $u(n-k)+k(m+u)=g$, then $g^{\prime}=(u+m, n-k)$ has to divide $g$, thus $m$ and $n$, thus $u$ and $k$, which gives $\left.g^{\prime}=1\right)$. As a consequence, there are exactly $g$ regular unstable bundles up to extended isomorphism.

Let us turn to subregular bundles. If $g=1$, there is always a $\mathbb{P}^{1}$-family of subregular bundles, up to extended isomorphism. This follows from an argument similar to that in the regular case. Namely, there is exactly one integer $k$, $0<k<n$, and an integer $u$ satisfying $u n+k m=2$. Similar to the arguments in the regular case, one checks either $(u, k)=2$ and $(u+m, n-k)=1$, or $(u, k)=1$ and $(u+m, n-k)=2$. These last conditions guarantee $\operatorname{dim} \operatorname{Aut}_{L_{k}}\left(\eta_{k}\right)=2$ (in obvious notation) from which our assertions follow. This family may be accompanied by an additional single subregular unstable bundle (up to extended isomorphism). This single bundle is then associated to a Levi subgroup $L_{3 k-n}$, where the number $k$ satisfies $0<k<n$, un $+k m=1$ and the inequality $n<3 k<2 n$.

Assume $g \geqslant 2$. Then there are $g-1$ distinct families of subregular unstable bundles parametrized by the elliptic curve $E$. This follows from an analysis of the unstable bundles associated with corank-2 Levi subgroups $L$. One can show that the Levi subgroups of type $L=L_{k, k+n / g}$, where $k$ satisfies $u n+k m=g$, and only those, give rise to unstable bundles $\xi$ with $\operatorname{dim} \operatorname{Aut}_{G}(\xi)^{+}=g+1$. They are most easily described in terms of the associated projective space bundles, i.e. by using a fractional line bundle

$$
\xi \quad \longleftrightarrow U_{0}^{m / n} \otimes\left(U_{1} \oplus U_{2} \oplus U_{3}\right) .
$$

Here, $U_{0}$ is a line bundle of degree 1 , and $U_{i}$ is a stable vector bundle of rank 
$n_{i}$ and degree $d_{i}$ for $i=1,2,3$, where

$$
\left(n_{1}, d_{1}\right)=(k, u), \quad\left(n_{2}, d_{2}\right)=(n / g,-m / g), \quad\left(n_{3}, d_{3}\right)=\left(k^{\prime}, u^{\prime}\right)
$$

with $k^{\prime}=n-(n / g)-k$ and $u n+k m=g, u^{\prime} n+k^{\prime} m=-g$. One easily verifies that these bundles satisfy $\operatorname{dim} \operatorname{Aut}_{L}\left(\xi_{L}\right)=2$.

If $g=2$ and $n>4$, then there is, up to extended isomorphism, an additional single subregular unstable bundle associated to one of the two Levi subgroups $L_{k}$ whose index $k$ satisfies $u n+k m=4$.

The existence of isolated subregular unstable bundles can be interpreted in terms of orbit closures inside the extended loop group $\hat{\mathcal{L}} G$ (cf. [13], [14]). In the above cases, they lead to simple elliptic singularities of type $\tilde{E}_{6}, g=1$, and $\tilde{D}_{5}, g=2$. The families of subregular unstable bundles are related to singularities of type $A_{\infty}$ and $D_{\infty}$ depending on the parametrization by either $E$ or $\mathbb{P}^{1}$.

(6.3) The CASE $B_{\ell}$. The adjoint group of type $B_{\ell}$ is isomorphic to the special orthogonal group $S O_{2 \ell+1}$. Its fundamental group is $\mathbb{Z} / 2 \mathbb{Z}$, and the reduced rank is $\ell_{\overline{1}}=\ell-1$. For the fundamental dominant weights, we have the following relations

$$
\left[\varpi_{k}\right]=\varpi_{k} \quad \text { for } \quad k<\ell \quad \text { and } \quad\left[\varpi_{\ell}\right]=2 \varpi_{\ell},
$$

leading, by Theorem 2.6, to the dimensions $\operatorname{dim} \operatorname{Aut}_{G}(\xi)^{+}$for maximal bundles of type $P_{k}$ as tabulated below. The structure of the Levi subgroups $L_{k}$ has already been discussed before in relation to $\operatorname{Spin}_{2 \ell+1}$, i.e. $L_{k}=$ $G L_{k} \times S O_{2(\ell-k)+1}$. Let us first consider the extremal case $k=\ell$, in which $L_{\ell}=G L_{\ell}$. Then $H_{\ell}=S L_{\ell}$ is simply connected (Situation I). The exact sequence

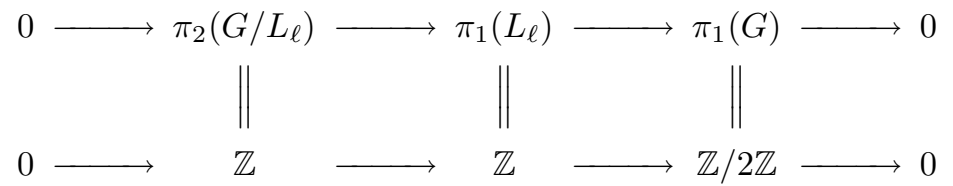

shows that $G L_{\ell}$-bundles $\eta$ of odd degree extend to $S O_{2 \ell+1}$-bundles $\xi$ of topological type $\gamma(\xi)=\overline{1}$. For bundles $\eta$ of degree 1 we get $\operatorname{dim}_{\operatorname{Aut}_{L_{\ell}}}(\eta)=1$. Assume now $k<\ell$. Then $\pi_{1}\left(H_{k}\right)=\mathbb{Z} / 2 \mathbb{Z}$ maps isomorphically to $\pi_{1}(G)$ (Situation II). For $L_{k}$-bundles $\eta$ of degree 1 , we have two choices according to the topological type of the $S O_{2(\ell-k)+1}$-component $\eta_{2}$. If $\eta_{2}$ is a proper $S O_{2(\ell-k)+1}$-bundle, then, as we have seen in the discussion of $\operatorname{Spin}_{2 \ell+1}$, the bundle $\eta$ itself reduces to the Spin-Levi subgroup $\tilde{L}_{k} \subset \operatorname{Spin}_{2 \ell+1}$. Thus $\eta_{2}$ has to be a topologically trivial bundle, now, reducing to $\operatorname{Spin}_{2(\ell-k)+1}$. Then $\operatorname{dim} \operatorname{Aut}_{S O_{2(\ell-k)+1}}\left(\eta_{2}\right)=\ell-k$ 
and $\operatorname{dim} \operatorname{Aut}_{L_{k}}(\eta)=\ell+1-k$. (In more geometric terms, the reason behind the above argument is that the Stiefel-Whitney class of the extended bundle $\eta$ is the sum of the individual Stiefel-Whitney classes of $\eta_{1}$ and $\eta_{2}$-in obvious notation-).

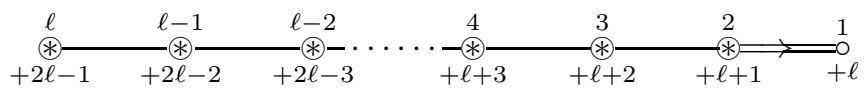

Up to extended isomorphism, we find one regular unstable bundle associated with the Levi subgroup $L_{\ell}$ and a $\mathbb{P}^{1}$-family of subregular unstable bundles associated with $L_{\ell-1}$.

(6.4) THE CASE $C_{\ell}$. The adjoint group of this type is $G=P S p_{2 \ell}=S p_{2 \ell} /\langle-1\rangle$ with fundamental group $\pi_{1}=\mathbb{Z} / 2 \mathbb{Z}$ and reduced rank $\ell_{\overline{1}}=m$, where either $\ell=2 m$ or $\ell=2 m+1$. For the fundamental dominant (co-)weights, the following relations hold

$$
\left[\varpi_{k}\right]=2 \varpi_{k} \text {, for } k \text { odd, }\left[\varpi_{k}\right]=\varpi_{k} \text {, for } k \text { even and }\left[\varpi_{k}^{\vee}\right]=\varpi_{k}^{\vee} \text {, for all } k \text {, }
$$

which, together with Theorem 2.6, gives the minimal values for $\operatorname{dim}_{\operatorname{Aut}_{G}}(\xi)^{+}$ of $G$-bundles $\xi$ with $\gamma(\xi)=\overline{1}$.

Concerning the structure of the Levi subgroups and semistable bundles of minimal degree, the parities of the involved numbers $\ell, k$, and $k / 2$, if $k$ is even, will play a role.

Let us first have a look at the Levi subgroups $L_{k}$ for odd $k$, in which case we have $\left\langle\left[\varpi_{k}\right], \varpi_{k}^{\vee}\right\rangle=2 k$. The group $L_{k}$ is the quotient of $G L_{k} \times S p_{2(\ell-k)}$ by the diagonal subgroup $\mu_{2}$ with simply connected semisimple part $H_{k}=S L_{k} \times$ $S p_{2(\ell-k)}$ (Situation I). Let $\eta$ be an $L_{k}$-bundle of minimal degree 1 . Then the topological class of its $\bar{H}_{k}$-extension $\bar{\eta}$ is a generator of $\pi_{1}\left(\bar{H}_{k}\right)=\mathbb{Z} / k \mathbb{Z} \times \mathbb{Z} / 2 \mathbb{Z}$. From that, we conclude $\operatorname{dim}_{\operatorname{Aut}_{L}}(\eta)=0+\ell^{\prime}+1=m+1-(k-1) / 2$, if $\ell$ is odd, and $\operatorname{dim} \operatorname{Aut}_{L_{k}}(\eta)=0+\ell^{\prime}+1=m-(k-1) / 2$, if $\ell$ is even.

We now turn to the case $k=2 s$ even. Again $L_{k}$ is the quotient of $G L_{k} \times$ $S p_{2(\ell-k)}$ by the diagonal subgroup $\mu_{2}$. However, this time, the semisimple part $H_{k}$ is not any more simply connected but isomorphic to the diagonal quotient $\left(S L_{k} \times S p_{2(\ell-k)}\right) / \mu_{2}$ with fundamental group $\pi_{1}\left(H_{k}\right)=\mathbb{Z} / 2 \mathbb{Z}$ (Situation II). By the general arguments in 6.1 , we can identify $\pi_{1}(L) \longrightarrow \pi_{1}(G)$ with the second projection

$$
\mathbb{Z} \varpi_{k}^{\vee} \times \pi_{1}(G) \longrightarrow \pi_{1}(G) .
$$

A semistable $L_{k}$-bundle $\eta$ of minimal degree 1 whose $G$-extension has nontrivial type in $\pi_{1}(G)$ has topological type $\gamma(\eta)=\left(\alpha_{k}^{\vee}, \overline{1}\right)$ in $\mathbb{Z} \alpha_{k}^{\vee} \times \mathbb{Z} / 2 \mathbb{Z}$. Let $\bar{\eta}$ be the semistable $\bar{H}$-bundle extension of $\eta$. We have to compute its topological 
class $\gamma(\bar{\eta})$ in $\pi_{1}(\bar{H})=\pi_{1}\left(P G L_{k}\right) \times \pi_{1}\left(P S p_{2(\ell-k)}\right)=\mathbb{Z} / k \mathbb{Z} \times \mathbb{Z} / 2 \mathbb{Z}$. Observe that under the projection $H \longrightarrow \bar{H}$, the group $\pi_{1}(H)$ is embedded diagonally into $\pi_{1}(\bar{H})=\mathbb{Z} / k \mathbb{Z} \times \mathbb{Z} / 2 \mathbb{Z}$, and the element $\alpha_{k}^{\vee} \in \pi_{1}(L)$ is mapped to the element $(\overline{1}, \overline{0})$ in $\mathbb{Z} / k \mathbb{Z} \times \mathbb{Z} / 2 \mathbb{Z}$. (This last fact is checked by a computation in the coweight lattice of type $C_{\ell}$ or, dually, in the weight lattice of type $B_{\ell}$.) Thus, the topological type $\gamma(\bar{\eta})$ is $(\overline{1}+\bar{s}, \overline{1}), s=k / 2$, yielding $\operatorname{dim}_{A^{\prime}}(\eta)=1+(m-$ $s)+1=m+2-s$, if $s$ is odd, resp. $\operatorname{dim}_{L} \operatorname{Aut}(\eta)=0+(m-s)+1=m+1-s$, if $s$ is even. Note that this reasoning covers also the case that $k=\ell$.

Here are diagrams representing that information graphically. Their left ends depend on the parity of $\ell$, and their right ends on the residue of $\ell$ modulo 4.

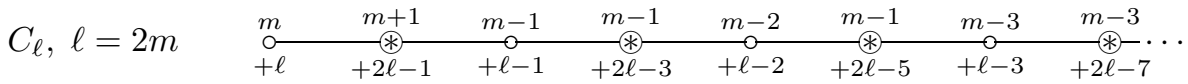

For $m$ even, the right end looks like:

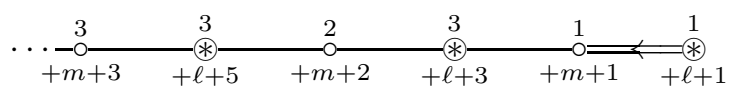

And for $m$ odd, we have:

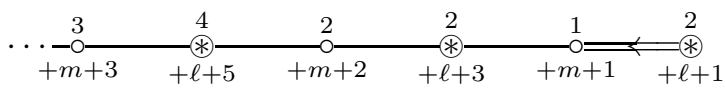

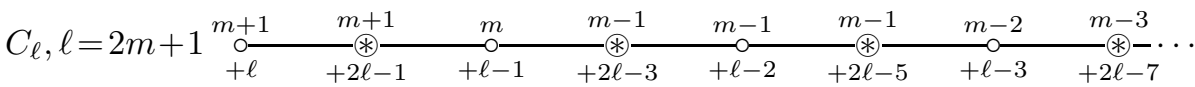

The right end for $m$ even:

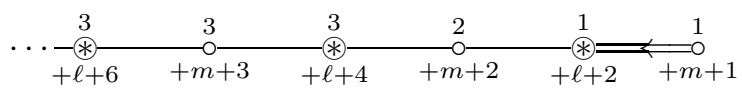

The right end for $m$ odd:

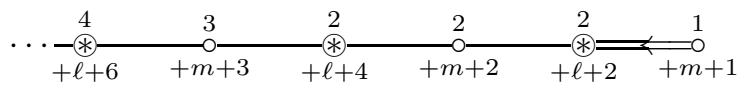

Here are some diagrams of low rank.

$$
\begin{aligned}
& C_{2}, P S p_{4} \\
& C_{3}, P S p_{6} \\
& C_{4}, P S p_{8} \\
& C_{5}, P S p_{10}
\end{aligned}
$$

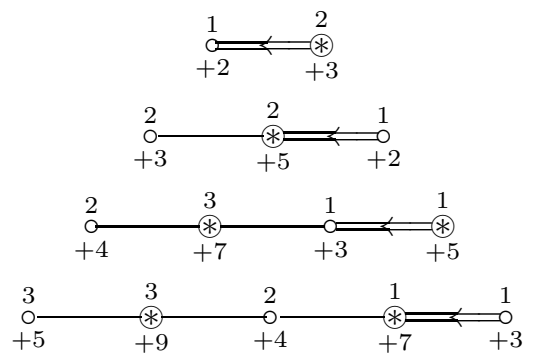


Up to extended isomorphism, we find one regular unstable bundle and a $\mathbb{P}^{1}$ family of subregular unstable bundles. They are associated with the Levi subgroups $L_{\ell-1}$ and $L_{\ell-3}$ if $\ell$ is even, resp. $L_{\ell}$ and $L_{\ell-2}$ if $\ell$ is odd.

(6.5) The CASE $D_{\ell}, G=S O_{2 \ell}$. This is one of the non simply connected groups of type $D_{\ell}$ with fundamental group $\mathbb{Z} / 2 \mathbb{Z}$ and reduced rank $\ell_{\overline{1}}=\ell-2$. It can be treated exactly as the odd orthogonal case. However, one has to take care of two extremal cases now, $k=\ell-1$ and $k=\ell$. The case $\ell=3$ which corresponds to $S O_{6} \cong S L_{4} / \mu_{2}$ can also be included.

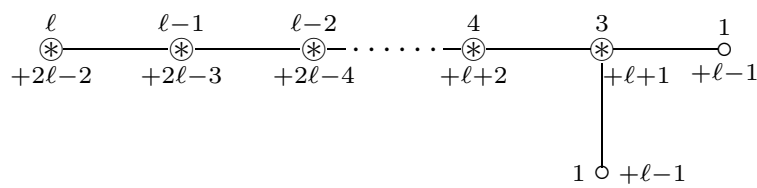

Up to extended isomorphism, we find two regular unstable bundle associated with the Levi subgroups $L_{\ell-1}$ and $L_{\ell}$. There is also a family of subregular unstable bundles $\xi$ parametrized by the elliptic curve $E$ and associated with the corank-2 Levi subgroup $L_{\ell-1, \ell}$. The orthogonal vector bundle associated to $\xi$ and the natural representation of $S_{2 \ell}$ has the form

$$
U_{\ell-1} \oplus\left(U_{\ell-1}\right)^{*} \oplus U_{1} \oplus\left(U_{1}\right)^{*},
$$

where $U_{\ell-1}$ is stable of rank $\ell-1$ and degree 1 , and where $U_{1}$ is a line bundle of degree 0 . In case $\ell=3$, this gives a family of subregular unstable $S L_{4} / \mu_{2^{-}}$ bundles of topological class $\overline{2}$.

(6.6) The CASE $D_{\ell}, \ell=2 m+1, G=P S O_{2 \ell}$. This is the other non simply connected, i.e. adjoint group of this type $D_{\ell}$ with fundamental group $\mathbb{Z} / 4 \mathbb{Z}=$ $P / Q$. As a generator $\overline{1}$ of $P / Q$ we fix the class of $\varpi_{\ell}$. Then $\overline{2}$ corresponds to the class of $\varpi_{1}$ and $\overline{3}$ to that of $\varpi_{\ell-1}$. Bundles of type $\gamma(\xi)=\overline{0}$ or $\overline{2}$ reduce to $\mathrm{Spin}_{2 \ell}$ or $\mathrm{SO}_{2 \ell}$ and have been dealt with before. Moreover, the outer automorphism exchanges the two types $\overline{1}$ and $\overline{3}$. We may therefore restrict to bundles of type $\overline{1}$. The reduced rank is $\ell_{\overline{1}}=m-1$.

Since $G$ is adjoint, all fundamental coweights $\varpi_{k}^{\vee}$ lie in $X_{*}(T)$. For the fundamental weights, we have

$\left[\varpi_{k}\right]=2 \varpi_{k}$, for $k<\ell-1$, odd, $\quad\left[\varpi_{k}\right]=\varpi_{k}$, for $k<\ell-1$, even and $\left[\varpi_{k}\right]=4 \varpi_{k}$, for $k=\ell-1$ and $k=\ell$.

For $k<\ell-1$, the $k$-th Levi subgroup $L_{k}$ is the quotient of $G L_{k} \times S O_{2(\ell-k)}$ by the diagonal subgroup $\mu_{2}$, and $L_{\ell-1} \cong L_{\ell} \cong G L_{\ell} / \mu_{2}$. The semisimple part 
$H_{k}$ has a fundamental group $\pi_{1}\left(H_{k}\right)$ of order $2,4,1$ in the respective cases. In particular, if $k<\ell-1$ is odd, we have $\pi_{1}\left(H_{k}\right)=\mathbb{Z} / 2 \mathbb{Z}$, canonically embedded into $\pi_{1}(G)=\mathbb{Z} / 4 \mathbb{Z}$ (Situation III). For even $k<\ell-1$, we get an isomorphism $\pi_{1}\left(H_{k}\right)=\pi_{1}(G)=\mathbb{Z} / 4 \mathbb{Z}$ (Situation II), and for $k=\ell-1$, $\ell$, we get $\pi_{1}\left(H_{k}\right)=0$ (Situation I).

Let us begin with the extremal case $k=\ell-1, \ell$ (Situation I). From the lattice interpretation of the surjective map

$$
\pi_{1}\left(L_{k}\right)=\mathbb{Z} \longrightarrow \mathbb{Z} / 4 \mathbb{Z}=\pi_{1}(G),
$$

we see that, for maximal $G$-bundles $\xi$ of type $\overline{1}$, we have to consider $L_{\ell}$-bundles $\eta$ of degree 1 or $L_{\ell-1}$-bundles $\eta$ of degree 3 , in case $\ell \equiv 1 \bmod 4$. If $\ell \equiv 3 \bmod$ 4 , the rôles of $\ell-1$ and $\ell$ are interchanged. According to the surjection

$$
\pi_{1}\left(L_{k}\right)=\mathbb{Z} \longrightarrow \mathbb{Z} / \ell \mathbb{Z} \cong \pi_{1}\left(\bar{H}_{k}\right) \cong \pi_{1}\left(P G L_{\ell}\right),
$$

the topological type of the $\bar{H}_{k}$-extension $\bar{\eta}$ will be $\overline{1}$ resp. $\overline{3}$. This leads to the value $\operatorname{dim} \operatorname{Aut}_{L_{k}}(\eta)=(3, \ell)$, which is 1 or 3 .

We now attack the next, still familiar, case $k=2 s<\ell-1$, (Situation II). Then $H_{k}$ is the diagonal quotient of $S L_{k} \times S O_{2(\ell-k)}$ by the diagonal $\mu_{2}$ with fundamental group $\pi_{1}\left(H_{k}\right)=\pi_{1}(G)=\mathbb{Z} / 4 \mathbb{Z}$. Thus, we may restrict to $L$ bundles $\eta$ of degree 1. Up to isomorphism of the factors, the surjection

$$
\pi_{1}(L)=\mathbb{Z} \alpha_{k}^{\vee} \times \mathbb{Z} / 4 \mathbb{Z} \longrightarrow \mathbb{Z} / k \mathbb{Z} \times \mathbb{Z} / 4 \mathbb{Z} \cong \pi_{1}\left(P G L_{k} \times P S O_{2(\ell-k)}\right)
$$

maps $\alpha_{k}^{\vee}$ to $(\overline{1}, \overline{2})$ and $(0, \overline{1})$ to $(\bar{s}, \overline{1})$. Thus, for $\bar{\eta}$ of topological type $\overline{1}$ or $\overline{3}$, we obtain $\operatorname{dim} \operatorname{Aut}_{L}(\eta)=((s+1,2 s)-1)+(m-s-1)+1=m-s-1+(s+1,2 s)$. Note that the value of $(s+1,2 s)$ is 1 or 2 according to $s$ being even or odd.

Finally, assume that $k$ is odd and $k<\ell-1$ (Situation III). Then we have $H_{k} \cong S L_{k} \times S O_{2(\ell-k)}$ with fundamental group $\pi_{1}\left(H_{k}\right)=\mathbb{Z} / 2 \mathbb{Z}$ injecting into the group $\pi_{1}(G)=\mathbb{Z} / 4 \mathbb{Z}$. From the surjective map

$$
\pi_{1}\left(L_{k}\right)=\mathbb{Z} \times \mathbb{Z} / 2 \mathbb{Z} \longrightarrow \mathbb{Z} / 4 \mathbb{Z}=\pi_{1}(G),
$$

we see that, for maximal $G$-bundles $\xi$ of type $\overline{1}$ or $\overline{3}$, it is sufficient to consider $L_{k}$-bundles $\eta$ of degree 1 . The homomorphism

$$
\pi_{1}(L)=\mathbb{Z} \times \mathbb{Z} / 2 \mathbb{Z} \longrightarrow \mathbb{Z} / k \mathbb{Z} \times \mathbb{Z} / 2 \mathbb{Z} \times \mathbb{Z} / 2 \mathbb{Z} \cong \pi_{1}\left(P G L_{k} \times P S O_{2(\ell-k)}\right)
$$

which maps the element $(0, \overline{1})$ to $(\overline{0}, \overline{1}, \overline{1})$, can be surjective only, if it maps $(1, \overline{0})$ to $(\overline{1}, \overline{1}, \overline{0})$ (up to possible isomorphism of the target). Thus, for $\operatorname{deg}(\eta)=1$ or 3 we get $\operatorname{dim} \operatorname{Aut}_{L_{k}}(\eta)=0+(\ell-k) / 2+1=m+1-(k-1) / 2$. 
The diagrams representing the dimension values start the same on their left end.

$$
\begin{aligned}
& P S O_{2 \ell}, \ell=2 m+1, \gamma(\xi)=\overline{1} .
\end{aligned}
$$

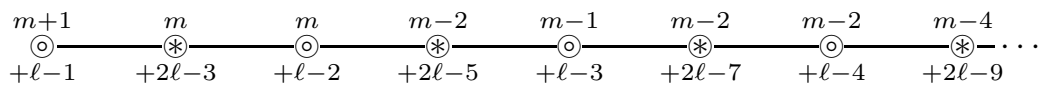

Diagrams for $\gamma(\xi)=\overline{3}$ are obtained by applying the diagram automorphism. The right end depends on the parity of $m$. Here is $m$ even.

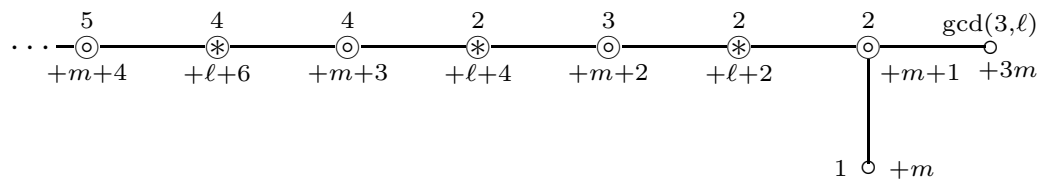

An example of smallest rank, $D_{5}, m=2$.

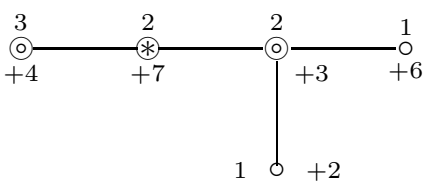

Here is $m$ odd.

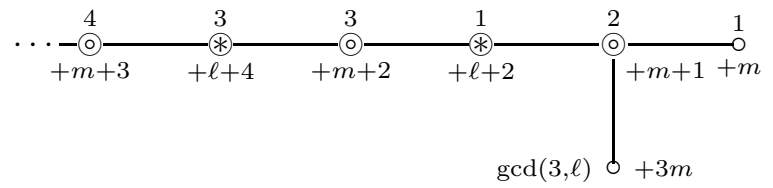

An example of smallest rank, $D_{7}, m=3$.

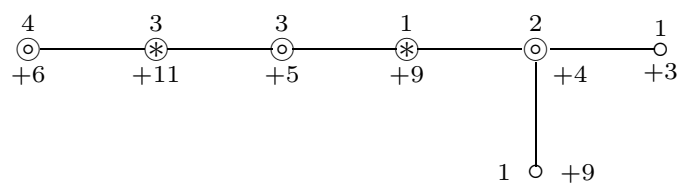

Up to extended isomorphism, we find one regular unstable bundle and a $\mathbb{P}^{1}$ family of subregular unstable bundles. They are associated with the Levi subgroups $L_{\ell}$ and $L_{\ell-2}$ if $m$ is even, resp. $L_{\ell-1}$ and $L_{\ell-2}$ if $m$ is odd.

(6.7) The CASE $D_{\ell}, \ell=2 m, G=P S_{2 \ell}$. This is the adjoint group of type $D_{\ell}$ with fundamental group $\mathbb{Z} / 2 \mathbb{Z} \times \mathbb{Z} / 2 \mathbb{Z}=P / Q$, generated by $(\overline{1}, \overline{0})=\varpi_{\ell-1}$ and $(\overline{0}, \overline{1})=\varpi_{\ell}$. The element $(\overline{1}, \overline{1})$ corresponds to the class of $\varpi_{1}$ in $P / Q$. 
Bundles of type $\gamma(\xi)=(\overline{0}, \overline{0})$ or $(\overline{1}, \overline{1})$ reduce to $\operatorname{Spin}_{2 \ell}$ or $S O_{2 \ell}$ and have been dealt with before. The other bundles reduce to the half-spin groups $\operatorname{Spin}_{2 \ell}^{+/-}$. However, this reduction has no effect on the dimension of their automorphism groups, and we therefore stay with the more accessible adjoint group. The outer automorphism exchanges the two types $(\overline{1}, \overline{0})$ and $(\overline{0}, \overline{1})$. We may therefore restrict to bundles of one of these types. The reduced rank is $\ell_{(\overline{1}, \overline{0})}=m$. Since $G$ is adjoint, all fundamental coweights $\varpi_{k}^{\vee}$ lie in $X_{*}(T)$. For the fundamental weights, we have

$$
\left[\varpi_{k}\right]=\varpi_{k}, \text { for } k \text { even, } k \neq \ell, \quad\left[\varpi_{k}\right]=2 \varpi_{k}, \text { for } k \text { odd, or } k=\ell .
$$

We start with the somewhat familiar Situation II, i.e. $k=2 s \neq \ell$. Then $L_{k}$ is the quotient of $G L_{k} \times S O_{2(\ell-k)}$ by the diagonal subgroup $\mu_{2}$, and $H_{k}=$ $S L_{k} \times S O_{2(\ell-k)} / \mu_{2}$ has fundamental group $\pi_{1}\left(H_{k}\right)=\pi_{1}(G)=\mathbb{Z} / 2 \mathbb{Z} \times \mathbb{Z} / 2 \mathbb{Z}$. Accordingly, we may restrict our attention to $L_{k}$-bundles $\eta$ of degree 1 . The surjection

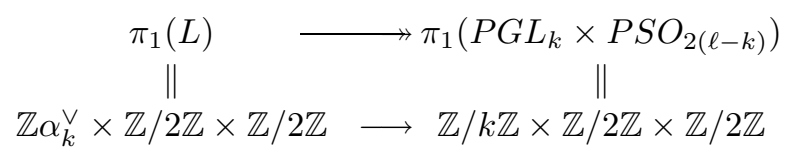

maps $\alpha_{k}^{\vee}$ to $(\overline{1}, \overline{1}, \overline{1})$ and, up to automorphism, $(0, \overline{1}, \overline{0})$ to $(\bar{s}, \overline{1}, \overline{0})$ and $(0, \overline{0}, \overline{1})$ to $(\bar{s}, \overline{0}, \overline{1})$. Assuming $\bar{\eta}$ to be of topological type $(\overline{1}, \overline{0})$ or $(\overline{0}, \overline{1})$, we obtain $\operatorname{dim} \operatorname{Aut}_{L}(\eta)=((s+1,2 s)-1)+(\ell-k) / 2+1=m-s+(s+1,2 s)$. Note that $(s+1,2 s)$ is 1 or 2 according to $s$ being even or odd.

Next, assume that $k=2 s+1$ is odd and $k<\ell-1$ (Situation III). Then $L_{k}$ is a diagonal quotient $G L_{k} \times S O_{2(\ell-k)} / \mu_{2}$ with semisimple part $H_{k} \cong S L_{k} \times S O_{2(\ell-k)}$. The fundamental group $\pi_{1}\left(H_{k}\right)=\mathbb{Z} / 2 \mathbb{Z}$ injects diagonally into $\pi_{1}(G)=\mathbb{Z} / 2 \mathbb{Z} \times \mathbb{Z} / 2 \mathbb{Z}$. From the surjective map

$$
\pi_{1}\left(L_{k}\right)=\mathbb{Z} \times \mathbb{Z} / 2 \mathbb{Z} \longrightarrow \mathbb{Z} / 2 \mathbb{Z} \times \mathbb{Z} / 2 \mathbb{Z}=\pi_{1}(G),
$$

we see that, for maximal unstable $G$-bundles $\xi$ of type $(\overline{1}, \overline{0})$ or $(\overline{0}, \overline{1})$, it is sufficient to consider $L_{k}$-bundles $\eta$ of degree 1 . The homomorphism

$$
\pi_{1}(L)=\mathbb{Z} \times \mathbb{Z} / 2 \mathbb{Z} \longrightarrow \mathbb{Z} / k \mathbb{Z} \times \mathbb{Z} / 4 \mathbb{Z} \cong \pi_{1}\left(P G L_{k} \times P S O_{2(\ell-k)}\right)
$$

maps the element $(0, \overline{1})$ to $(\overline{0}, \overline{2})$. Because of surjectivity, it must map the element $(1, \overline{0})$ to $(\overline{1}, \overline{1})$, up to isomorphism of the target. Thus, for the relevant $L_{k}$-bundles $\eta$ we get $\operatorname{dim} \operatorname{Aut}_{L_{k}}(\eta)=0+((\ell-k-1) / 2-1)+1=m-1-s$.

Finally, let us treat the extremal cases $k=\ell-1, \ell$ (Situation III) (by symmetry, it is sufficient to look at $k=\ell$, but then we have to allow both 
relevant types of the bundle $\xi$ ). In the lattice interpretation of the surjective map

$$
\pi_{1}\left(L_{\ell}\right)=\mathbb{Z} \longrightarrow \mathbb{Z} / 2 \mathbb{Z} \times \mathbb{Z} / 2 \mathbb{Z}=\pi_{1}(G),
$$

the class of $\varpi_{1}$ (chosen generator of $\mathbb{Z}$ ) is mapped to $(\overline{1}, \overline{1})$, and the torsion element $(0, \overline{1})$, i.e. the class of $(\ell-2) / 2 \varpi_{1}-\varpi_{\ell-1}$, is mapped to $(\overline{1}, \overline{0})$, if $m=\ell / 2$ is odd, and to $(\overline{0}, \overline{1})$, if $m=\ell / 2$ is even. An $L_{\ell^{-}}$bundle $\eta$ of minimal positive degree will thus extend to a $G$-bundle $\xi$ of type $\gamma(\xi)=(\overline{1}, \overline{0})$ exactly when its class in $\pi_{1}\left(L_{\ell}\right)$ is $(1, \overline{1}), m$ even, or $(2, \overline{1}), m$ odd. We will have $\gamma(\xi)=(\overline{0}, \overline{1})$ exactly when $\gamma(\eta)=(1, \overline{1}), m$ odd, or $(2, \overline{1}), m$ even.

With respect to the same generators of $\pi_{1}\left(L_{\ell}\right)$ and the generating class $\varpi_{1}$ of $\pi_{1}(\bar{H})$, the map

$$
\pi_{1}\left(L_{\ell}\right)=\mathbb{Z} \longrightarrow \mathbb{Z} / \ell \mathbb{Z} \cong \pi_{1}(\bar{H}) \cong \pi_{1}\left(P G L_{\ell}\right)
$$

sends $\varpi_{1}$ to $\overline{1}$ and $(0, \overline{1})$ to $\bar{\ell} / 2$. In particular, the elements $(1, \overline{1}),(2, \overline{1})$, are mapped to $\bar{m}+\overline{1}$, resp. $\bar{m}+\overline{2}$. The corresponding dimensions $\operatorname{dim}_{A^{\prime}} \operatorname{dut}_{L}(\eta)$ are $(m+1,2 m)$, resp. $(m+2,2 m)$. If $m$ is odd, these are 2 , resp. 1 . If $m=2 s$ is even, they are 1 resp. 2 or 4 , depending on $s$ being even or odd.

The diagrams representing the dimension values start the same on their left end. $P S O_{2 \ell}, \ell=2 m, \gamma(\xi)=(\overline{1}, \overline{0})$.

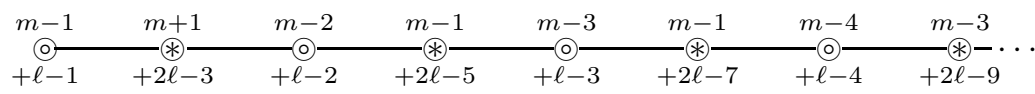

The right end depends on the parity of $m$. Here is $m$ even.

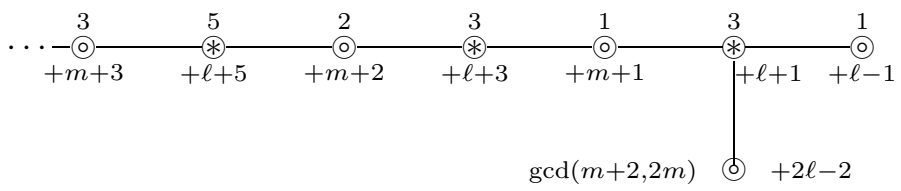

An example of smallest rank, $D_{4}, m=2$. By triality, this is equivalent to the case of $\mathrm{SO}_{8}$.

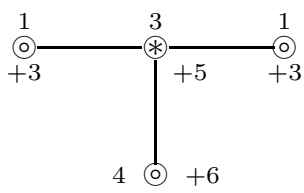

Here is $m$ odd.

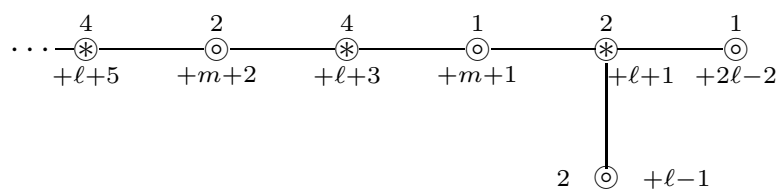


An example of smallest rank, $D_{6}, m=3$.

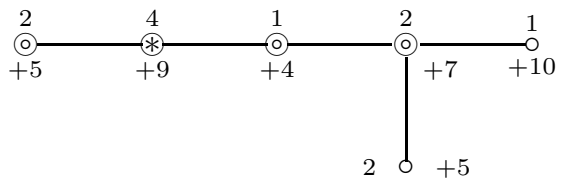

In the following, we shall exclude the case $m=2$, i.e. $D_{4}$, which has been dealt with already $\left(\mathrm{SO}_{8}\right)$. Up to extended isomorphism, we find one regular unstable bundle and a $P^{1}$-family of subregular unstable bundles. They are associated with the Levi subgroups $L_{\ell-3}$ and $L_{\ell-5}$.

(6.8) The CASE $E_{6}$. The adjoint group $G=E_{6}^{a d}$ of type $E_{6}$ has fundamental group $\pi_{1}=\mathbb{Z} / 3 \mathbb{Z}$ and reduced rank $\ell_{\overline{1}}=\ell_{\overline{2}}=2$. The set of indices $k=1, \ldots, 6$ of $\Delta$ decomposes into two parts, $I_{I}=\{1,3,5,6\}$, which contains those $k$ with $\left[\varpi_{k}\right]=3 \varpi_{k}$, and $I_{I I}=\{2,4\}$, which contains those $k$ with $\left[\varpi_{k}\right]=\varpi_{k}$. These cases will also correspond to the two situations $\pi_{1}\left(H_{k}\right)=0$ resp. $\pi_{1}\left(H_{k}\right)=$ $\pi_{1}(G)=\mathbb{Z} / 3 \mathbb{Z}$. The outer automorphism of $G$ of order 2 interchanges the two non trivial center elements of $\tilde{G}$ and accordingly $G$-bundles of topological types $\overline{1}$ and $\overline{2}$. It is therefore sufficient to look at bundles of type $\overline{1}$. We shall fix the isomorphism $\pi_{1}(G)=P / Q$ and the element $\overline{1}$ as the class of the weight $\varpi_{1} \in P / Q$ (here we have identified the weight and coweight lattices, which we shall also do below).

Let us first study the Situation I which essentially means $k=1,3$, the cases $k=5,6$ being symmetric to those by the outer automorphism. We have $H_{1}=S_{p i n} 10$ and $H_{3}=S L_{2} \times S L_{5}$ with respective modules $\mathfrak{g}(-1)$ isomorphic to $\Delta_{+}$, one of the half-spin representations, and $\mathbb{C}^{2} \otimes \bigwedge^{3} \mathbb{C}^{5}$. This leads to the symbol $[3]_{4}$ for $L_{1}$ resp. $[5,8]_{10}$ for $L_{3}$. If $\eta$ is an $L$-bundle of degree 1 this gives the value 2 resp. 1 for $\operatorname{dim}_{A^{\prime}}(\eta)$. If $\operatorname{deg}(\eta)=2$ we get the value 4 resp. 2. The classes of $\varpi_{k} / d, k=1,3,5,6$ in $P / Q$ are $\varpi_{1}, 2 \varpi_{1}, \varpi_{1}, 2 \varpi_{1}$ (note that $d \equiv 1(\bmod 3)$ in all cases). Thus, extending $\eta$ to a $G$-bundle $\xi$ gives topological type $\gamma(\xi)=\overline{1}$ if $\operatorname{deg}(\eta)=1,2,1,2$ for $k=1,3,5,6$.

The cases $k=2,4$ belong to Situation II. Let $k=2$. We have $H_{2}=S L_{6} / \mu_{3}$ with fundamental group $\mathbb{Z} / 3 \mathbb{Z}$, acting on $\mathfrak{g}(-1)$ by means of $\bigwedge^{3} \mathbb{C}^{6}$. The value of $d=\left\langle\varpi_{2}, \varpi_{2}\right\rangle$ is 2 , and the map

$$
\pi_{1}\left(L_{2}\right)=\mathbb{Z} \alpha_{2} \times \pi_{1}\left(H_{2}\right) \longrightarrow \pi_{1}\left(\bar{H}_{2}\right)=\pi_{1}\left(P G L_{6}\right)
$$

can be written explicitly as follows

$$
\mathbb{Z} \times \mathbb{Z} / 3 \mathbb{Z} \longrightarrow \mathbb{Z} / 6 \mathbb{Z}, \quad(m, \bar{n}) \mapsto(3 \bar{m}+2 \bar{n}) .
$$


As a consequence, for any $L_{2}$-bundle of degree 1 with topologically non-trivial extension to $G$, we get $\operatorname{dim} \operatorname{Aut}_{L_{2}}(\eta)=1$.

Let $k=4$. Here, $H_{4}=\left(S l_{3} \times S L_{2} \times S L_{3}\right) / \mu_{3}$ with fundamental group $\mathbb{Z} / 3 \mathbb{Z}$. It acts on $\mathfrak{g}(-1)$ by means of the representation $\mathbb{C}^{3} \otimes \mathbb{C}^{2} \otimes \mathbb{C}^{3}$. We have $d=\left\langle\varpi_{4}, \varpi_{4}\right\rangle=6$, and the map

$$
\pi_{1}\left(L_{4}\right)=\mathbb{Z} \alpha_{4} \times \pi_{1}\left(H_{4}\right) \longrightarrow \pi_{1}\left(\bar{H}_{4}\right)=\pi_{1}\left(P G L_{3}\right) \times \pi_{1}\left(P G L_{2}\right) \times \pi_{1}\left(P G L_{3}\right)
$$

can be written explicitly as follows

$$
\mathbb{Z} \times \mathbb{Z} / 3 \mathbb{Z} \longrightarrow \mathbb{Z} / 3 \mathbb{Z} \times \mathbb{Z} / 2 \mathbb{Z} \times \mathbb{Z} / 3 \mathbb{Z}, \quad(m, \bar{n}) \mapsto(\bar{m}+\bar{n}, \bar{m}, \bar{m}+2 \bar{n}) .
$$

For all $L_{4}$-bundles of degree 1 with topologically non-trivial extension to $G$, this gives $\operatorname{dim} \operatorname{Aut}_{L_{4}}(\eta)=3$.

Here is a diagram collecting the numerical values for all $P_{k}$-maximal unstable $G$-bundles $\xi$ of topological type $\gamma(\xi)=\overline{1}$. The diagram for topological type $\gamma(\xi)=\overline{2}$ is obtained by applying the diagram symmetry.

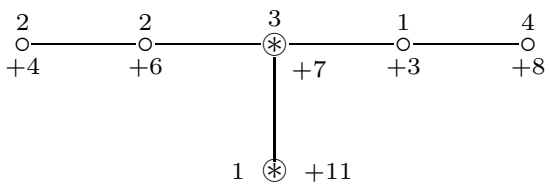

Up to extended isomorphism, we find one regular unstable bundle and a $P^{1}$ family of subregular unstable bundles. They are associated with the Levi subgroups $L_{5}$ and $L_{1}$. Of course, when changing from $\gamma(\xi)=\overline{1}$ to $\gamma(\xi)=\overline{2}$, these Levi subgroups are replaced by $L_{3}$ and $L_{6}$.

(6.9) The CASE $E_{7}$. The adjoint group $G=E_{7}^{a d}$ of type $E_{7}$ has fundamental group $\pi_{1}=\mathbb{Z} / 2 \mathbb{Z}$ and reduced rank $\ell_{\overline{1}}=4$. Again, the set of indices $k=$ $1, \ldots, 7$ decomposes into two parts, $I_{I}=\{2,5,7\}$, which contains those $k$ with $\left[\varpi_{k}\right]=2 \varpi_{k}$ and $\pi_{1}\left(H_{k}\right)=0$, and $I_{I I}=\{1,3,4,6\}$, which contains those $k$ with $\left[\varpi_{k}\right]=\varpi_{k}$ and $\pi_{1}\left(H_{k}\right)=\pi_{1}(G)=\mathbb{Z} / 2 \mathbb{Z}$.

The Situation I arises with $k=2,5,7$. We have $H_{2}=S L_{7}, H_{5}=S L_{5} \times$ $S L_{2}$ and $H_{7}=E_{6}^{s c}$ acting on $\mathfrak{g}(-1)$ by a representation isomorphic to $\bigwedge^{3} \mathbb{C}^{7}$ resp. $\wedge^{3} \mathbb{C}^{5} \otimes\left(\mathbb{C}^{3}\right)^{*}$ resp. $\mathbb{C}^{27}$. This leads to the symbols $[2]_{7}$, resp. $[3,10]_{15}$, resp. $[2]_{3}$. If $\eta$ is a semistable $L$-bundle of degree 1 , this gives the value 1 , resp. 1 , resp. 3 for $\operatorname{dim}_{\operatorname{Aut}_{L}}(\eta)$.

Let us now treat $k \in I_{I I}=\{1,3,4,6\}$. The group $H_{1}$ is isomorphic to the half-spin group $\operatorname{Spin}_{12}^{+}$with fundamental group $\mathbb{Z} / 2 \mathbb{Z}$. It acts faithfully on $\mathfrak{g}(-1)$ by means of its half-spin representation. We have $d=\left\langle\varpi_{1}, \varpi_{1}\right\rangle=2$, 
$\bar{H}_{1}=P S O_{12}$, and the map

$$
\pi_{1}\left(L_{1}\right)=\mathbb{Z} \alpha_{1} \times \pi_{1}\left(H_{1}\right) \longrightarrow \pi_{1}\left(\bar{H}_{1}\right)=\pi_{1}\left(P S O_{12}\right)
$$

can be written explicitly as follows

$$
\mathbb{Z} \times \mathbb{Z} / 2 \mathbb{Z} \longrightarrow \mathbb{Z} / 2 \mathbb{Z} \times \mathbb{Z} / 2 \mathbb{Z}, \quad(m, \bar{n}) \mapsto(\bar{m}, \bar{n}) .
$$

For an $L_{1}$-bundle $\eta$ of degree 1 with non-trivial extension to $G$, this gives the dimension 5 for $\operatorname{Aut}_{L_{1}}(\eta)$.

The group $H_{3}$ is isomorphic to $S L_{2} \times S L_{6} / \mu_{2}$ with fundamental group $\mathbb{Z} / 2 \mathbb{Z}$. It acts faithfully on $\mathfrak{g}(-1)$ by means of the representation $\mathbb{C}^{2} \otimes \bigwedge^{2} \mathbb{C}^{6}$. The order of intersection with $S_{3}$ is $d=\left\langle\varpi_{3}, \varpi_{3}\right\rangle=6, \bar{H}_{3}=P G L_{2} \times P G L_{6}$, and the map

$$
\pi_{1}\left(L_{3}\right)=\mathbb{Z} \alpha_{3} \times \pi_{1}\left(H_{3}\right) \longrightarrow \pi_{1}\left(\bar{H}_{3}\right)=\mathbb{Z} / 2 \mathbb{Z} \times \mathbb{Z} / 6 \mathbb{Z}
$$

can be written explicitly as follows

$$
\mathbb{Z} \times \mathbb{Z} / 2 \mathbb{Z} \longrightarrow \mathbb{Z} / 2 \mathbb{Z} \times \mathbb{Z} / 6 \mathbb{Z}, \quad(m, \bar{n}) \mapsto(\bar{m}, 2 \bar{m}+3 \bar{n}) .
$$

For an $L_{3}$-bundle $\eta$ of degree 1 with non-trivial extension to $G$, this gives the dimension 1 for $\operatorname{Aut}_{L_{3}}(\eta)$.

The group $H_{4}$ is isomorphic to $S L_{3} \times\left(S L_{2} \times S L_{4}\right) / \mu_{2}$ with fundamental group $\mathbb{Z} / 2 \mathbb{Z}$. It acts faithfully on $\mathfrak{g}(-1)$ by means of the representation $\mathbb{C}^{3} \otimes$ $\mathbb{C}^{2} \otimes \mathbb{C}^{4}$. The order of intersection with $S_{4}$ is $d=\left\langle\varpi_{4}, \varpi_{4}\right\rangle=12, \bar{H}_{4}=$ $P G L_{3} \times P G L_{2} \times P G L_{4}$, and the map

$$
\pi_{1}\left(L_{4}\right)=\mathbb{Z} \alpha_{4} \times \pi_{1}\left(H_{4}\right) \longrightarrow \pi_{1}\left(\bar{H}_{4}\right)=\mathbb{Z} / 3 \mathbb{Z} \times \mathbb{Z} / 2 \mathbb{Z} \times \mathbb{Z} / 4 \mathbb{Z}
$$

can be written explicitly as follows

$$
\mathbb{Z} \times \mathbb{Z} / 2 \mathbb{Z} \longrightarrow \mathbb{Z} / 3 \mathbb{Z} \times \mathbb{Z} / 2 \mathbb{Z} \times \mathbb{Z} / 4 \mathbb{Z}, \quad(m, \bar{n}) \mapsto(\bar{m}, \bar{m}+\bar{n}, \bar{m}+2 \bar{n}) .
$$

For an $L_{4}$-bundle $\eta$ of degree 1 with non-trivial extension to $G$, this gives the dimension 2 for $\operatorname{Aut}_{L_{4}}(\eta)$.

Finally, the group $H_{6}$ is isomorphic to $\left(\operatorname{Spin}_{10} \times S L_{2}\right) / \mu_{2}$ with fundamental group $\mathbb{Z} / 2 \mathbb{Z}$. It acts faithfully on $\mathfrak{g}(-1)$ by means of the tensor product representation $\Delta^{+} \otimes \mathbb{C}^{2}$, where $\Delta^{+}$is a half-spin module. The order of intersection with $S_{6}$ is $d=\left\langle\varpi_{6}, \varpi_{6}\right\rangle=4, \bar{H}_{6}=P S O_{10} \times P G L_{2}$, and the map

$$
\pi_{1}\left(L_{6}\right)=\mathbb{Z} \alpha_{6} \times \pi_{1}\left(H_{6}\right) \longrightarrow \pi_{1}\left(\bar{H}_{6}\right)=\mathbb{Z} / 4 \mathbb{Z} \times \mathbb{Z} / 2 \mathbb{Z}
$$


can be written explicitly as follows

$$
\mathbb{Z} \times \mathbb{Z} / 2 \mathbb{Z} \longrightarrow \mathbb{Z} / 4 \mathbb{Z} \times \mathbb{Z} / 2 \mathbb{Z}, \quad(m, \bar{n}) \mapsto(\bar{m}+2 \bar{n}, \bar{m}+\bar{n}) .
$$

For an $L_{6}$-bundle $\eta$ of degree 1 with non-trivial extension to $G$, this gives the dimension 3 for $\operatorname{Aut}_{L_{6}}(\eta)$.

The following diagram collects the dimension values for all $P_{k}$-maximal unstable $E_{7}^{a d}$-bundles $\xi$ of topological type $\gamma(\xi)=\overline{1}$.

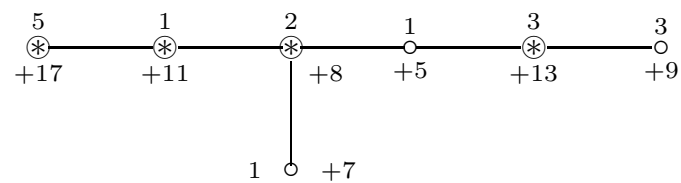

Up to extended isomorphism, we find one regular and one subregular unstable bundle. They are associated with the Levi subgroups $L_{5}$ and $L_{2}$.

(6.10) Remark. One can investigate the orbit closure singularity arising in this situation as described in [13], [14]. One finds a simply elliptic singularity of type $\tilde{E}_{6}$ which is unfolded semiuniversally with respect to a natural $\mathbb{Z}_{2}$ symmetry. This deformation has been studied before by Yano [28]. It is a free deformation of type $F_{4}^{\vee}$ in his terminology.

\section{References}

[1] Atiyah, M. F., Vector bundles over an elliptic curve, Proc. London Math. Soc., 7 (1957), 414-452.

[2] Atiyah, M. F. and Bott, R., The Yang-Mills equations over Riemann surfaces, Phil. Transact. Royal Soc. London Ser. A, 308 (1982), 523-615.

[3] Borel, A., Linear algebraic groups, 2nd ed., Graduate Texts in Math. 126, Springer, Berlin, Heidelberg, New York, 1996.

[4] Borel, A., Friedman, R. and Morgan, J., Almost commuting elements in compact Lie groups, eprint math. GR/9907007.

[5] Bourbaki, N., Groupes et algèbres de Lie, Chap., IV, V, VI, Hermann, Paris, 1968.

[6] Brieskorn, E., Singular elements of semisimple algebraic groups, Actes Congr. Int. Math., Nice, 2 (1970), 279-284.

[7] Etingof, P. and Frenkel, I. B., Central extensions of current groups in two dimensions, Comm. Math. Phys., 165 (1994), 429-444.

[8] Etingof, P. and Khesin, B. A., Affine Gelfand-Dickey brackets and holomorphic vector bundles, Geom. Funct. Anal., 4 (1994), 399-423.

[9] Friedman, R. and Morgan, J., Holomorphic principal bundles over elliptic curves, Duke eprint math. AG/9811130.

[10] Friedman, R., Morgan, J. and Witten, E., Vector bundles and F-theory, Comm. Math. Phys., 187 (1997), 679-743. 
[11] Grothendieck, A., A general theory of fiber spaces with structure sheaf, University of Kansas Report, Number 4 (1955).

[12] Harder, G. and Narasimhan, M. S., On the cohomology groups of moduli spaces of vector bundles over curves, Math. Ann., 212 (1975), 215-248.

[13] Helmke, S., Loop groups and elliptic singularities, Proc. Kinosaki Conf. on Algebraic Geometry, Nov. 1998, 98-105.

[14] Helmke, S. and Slodowy, P., Loop groups, principal bundles over elliptic curves and elliptic singularities, Annual Meeting of the Math. Soc. of Japan, Hiroshima, Sept. 1999, Abstracts, Section Infinite-dimensional Analysis, 67-77.

[15] Humphreys, J., Linear algebraic groups, Grad. Texts in Math. 21, Springer, Berlin, Heidelberg, New York, 1975.

[16] Kac, V. G., Infinite dimensional Lie algebras, Cambridge University Press, 1990.

[17] Kobayashi, S. Differential geometry of complex vector bundles, Publ. Math. Soc. of Japan 15, Iwanami Shoten and Princeton University Press, Tokyo, Princeton, 1987.

[18] Looijenga, E. J., Root systems and elliptic curves, Invent. Math., 38 (1976), 17-32.

[19] Mumford, D., Fogarty, J. and Kirwan, F., Geometric invariant theory 3rd ed., Grundlehren Math. Wiss. 333, Springer, Berlin, Heidelberg, New York, 1994.

[20] Ramanathan, A., Stable principal bundles on a compact Riemann surface, Math. Ann., 213 (1975), 129-152.

[21] Ramanan, S. and Ramanathan, A., Some remarks on the instability flag, Tohoku Math. J., 36 (1984), 269-291.

[22] Saito, K., Einfach elliptische Singularitäten, Invent. Math., 23 (1974), 289-325.

[23] Saito, K. and Yoshii, D., Extended affine root systems IV (Simply-laced elliptic Lie algebras), Publ. RIMS, Kyoto Univ., 36 (2000), 385-422.

[24] Schweigert, C., On moduli spaces of flat connections with non-simply connected structure group, Nucl. Phys. B, 492 (1997), 743-755.

[25] Shatz, S. S., The decomposition and specialization of algebraic families of vector bundles, Compositio Math., 35 (1977), 163-187.

[26] Slodowy, P., Simple Singularities and Simple Algebraic Groups, Springer Lecture Notes in Math., 815, Springer, 1980.

[27] Springer, T. A., Linear algebraic groups, 2nd ed., Prog. Math., 9, Birkhäuser, Basel, Boston, 1998.

[28] Yano, T., Free deformations of simply elliptic singularities, Sci. Rep. Saitama Univ., 9 (1980), 71-79. 